\title{
Composición y abundancia taxonómica del zooplancton desde el Pacífico Sur hasta la Península Antártica 2016-2017
}

\section{Zooplankton composition and taxonomic abundance from South Pacific until Antarctic Peninsula 2016-2017}

\author{
Luz Helena Mojica López y Andrés Franco-Herrera \\ (D) 0000-0003-1942-0710 (D) $0000-0002-9809-8151$ \\ Universidad de Bogotá Jorge Tadeo Lozano, Facultad de Ciencias Naturales e Ingeniería, Programa de Biología Marina, Carrera 2 No. 11-68, El \\ Rodadero,Santa Marta, Magdalena,Colombia.luz.mojica@utadeo.edu.co; andres.franco@utadeo.edu.co
}

\section{RESUMEN}

$\mathrm{C}$

on el propósito de contribuir al conocimiento del zooplancton asociado a las masas de agua presentes a lo largo de un gradiente latitudinal desde Perú hasta la Península Antártica, se obtuvieron 14 muestras con una red cónica de ojo de malla de $250 \mu \mathrm{m}$ y $60 \mathrm{~cm}$ de diámetro de boca, desde 0 a 200 m de profundidad, en el marco de la III Expedición Científica de Colombia a la Antártica "Almirante Padilla" (Verano Austral 2016-2017). Los resultados permitieron determinar un total de 75 familia/morfotipos (f/m), pertenecientes a 32 órdenes. Los órdenes Calanoida (20\%) y Perciformes (12\%) predominaron en este estudio, mientras que los huevos y larvas de peces, junto con bivalvos, cipris, salpas, entre otros organismos gelatinosos, aportaron $54 \%$ de importancia de la abundancia total. Con relación a la densidad de organismos, las familias de la subclase Copepoda, Calanidae, Paracalanidae, Clausocalanidae, Oncaeidae y Eucalanidae fueron las más representativas del muestreo.

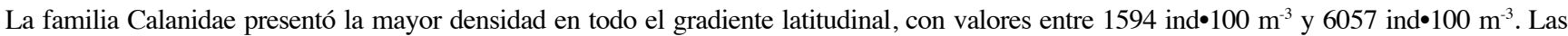
densidades más altas de zooplánctones fueron para el Pacífico Sur, donde se encontraron diferencias respecto a las densidades en las estaciones de la Antártica (Prueba Mood 1,4 valor-p de 0,24 con un $\alpha$ 0,05 y 95\% de confianza). Al realizar análisis multivariados se muestra similitud alta entre estaciones con relación a las abundancias del zooplancton en este estudio y se encontró una correlación de Pearson de 0,95, con esto se puede concluir que si existe una conectividad ecológica entre la comunidad del mesozooplancton a lo largo del Pacífico sur, lo cual se atribuye a el sistema de corrientes que juega un papel importante en la distribución de esta comunidad, así como eventos de afloramientos de aguas profundas que permiten encontrar familias cosmopolitas.

PALABRAS CLAVE: Zooplancton, Antártica, Pacífico sur, Conectividad.

\section{ABSTRACT}

$\mathrm{W}$ ith the purpose of contributing to the knowledge of the zooplankton associated with the water masses present along a latitudinal gradient from Peru to the Antarctic Peninsula, 14 samples were obtained, with a conical net of $250 \mu \mathrm{m}$ mesh size and $60 \mathrm{~cm}$ of mouth diameter, towed from 0 to $200 \mathrm{~m}$ water depth, within the III Scientific Expedition of Colombia to Antarctica "Almirante Padilla" (Austral Summer 2016-2017). The results allowed to determine a total of 75 family/morphotypes ( $\mathrm{f} / \mathrm{m}$ ), belonging to 32 orders. The orders Calanoida (20\%) and Perciformes (12\%) predominated in this study. Fish eggs and larvae, in addition to bivalves, and cypris, salps and other gelatinous organisms contributed with 54\% of importance. In relation to density, the families of the subclass Copepoda, Calanidae, Paracalanidae, Clausocalanidae, Oncaeidae and Eucalanidae were the most representative. Calanidae family showed the highest density across the latitudinal gradient, with values between 1594 ind $\bullet 100 \mathrm{~m}^{-3}$ and 6057 ind $\bullet 100 \mathrm{~m}^{-3}$. The highest densities of zooplankton were for the South Pacific, where differences were found with respect to the densities in the Antarctic stations (Mood: 1.4 p-value: $0.24 \alpha: 0.05$ and 95\% confidence). However, the cluster analysis showed an association between stations in relation to abundance of zooplankton in this study and found a Pearson correlation of 0.95 with this it can be concluded that if there is a ecological connectivity between the mesozooplankton community along the South Pacific, which is attributed to the system of currents that plays an important role in the distribution of this community, as well as events of deep water upwellings that allow to find cosmopolitan families.

KEYWORDS: Zooplankton, Antarctica, South Pacific, Connectivity. 


\section{INTRODUCCIÓN}

Los organismos planctónicos interactúan con diversos factores bióticos y abióticos que se encuentran en el ambiente marino y su estructura depende de los mismos. El zooplancton está compuesto por organismos que se distribuyen en toda la columna de agua y se convierte en clave de enlace dentro de las redes alimenticias pelágicas, en vista de que al agrupar el segundo nivel trófico, transfiere la energía y/o productividad primaria a los niveles superiores (González, 1998). La mayoría de los integrantes del zooplancton suelen considerarse como indicadores biológicos, por la capacidad que tienen algunos en adaptarse en ambientes hostiles de temperatura y salinidad (Fernández, 2015), también se consideran importantes en las pesquerías, debido a que las larvas de peces presentes en el zooplancton y sus ciclos biológicos resultan ser indicadores del reclutamiento y producción pesquera, así como también indicadores de tendencias de variación ambiental y afectaciones por contaminación (Ramírez y Mianzan, 2011; Fernández, 2015).

Los copépodos son probablemente los organismos más numerosos y diversos en los ambientes acuáticos, aportan la mayor biomasa, entre 40 y $98 \%$ de la densidad total del mesozooplancton en el océano Pacífico suelen ser dominantes (Pakhomov et al., 2000; Bernard y Froneman, 2002; Cabal et al., 2002; López, 2012; Jaimes y López, 2013; López y Mojica, 2015; Dorado-Roncancio, 2018). Las principales familias en la Antártica están representadas por Oithonidae, Oncaeidae, Pseudocalanidae, Calanidae y Metrididae (Cabal et al., 2002). Algunas especies de copépodos han mostrado respuestas a estímulos fóticos y otros aspectos de la biología básica que involucran comportamientos visualmente ontogénicos durante las migraciones verticales; estos comportamientos son impulsados por los ojos de los copépodos, incluidos los ojos naupliarios y los órganos de Gicklhorn (función fotoreceptora encontrados en los géneros Calanus y Euchaeta), que varían ampliamente en estructura y función entre las especies (Buskey et al., 2012). Sin embargo, su distribución en las masas de agua está estrechamente relacionada por el patrón de circulación local y regional, así como procesos oceanográficos a gran escala como eventos El Niño Oscilación del Sur-ENOS, que determinan características fisicoquímicas y biológicas en la columna de agua (López, 2008; Steenbeek et al., 2014).

\section{INTRODUCTION}

Planktonic organisms interact with various biotic and abiotic factors found in the marine environment on which their structure depends. Zooplankton is composed of organisms that are distributed throughout the water column and becomes a key link within the pelagic food webs, given that by grouping the second trophic level, it transfers energy and/or primary productivity to higher levels (González, 1998). The majority of zooplankton components are usually considered biological indicators, due to the capacity that some have to adapt to hostile environments of temperature and salinity (Fernández, 2015). They are also considered important in fisheries, because fish larva present in zooplankton and their biological cycles are indicators of fishery recruitment and production, as well as of trends in environmental variation and pollution impacts (Ramírez and Mianzan, 2011; Fernández, 2015).

Los copépodos son probablemente los organismos más numerosos y diversos en los ambientes acuáticos, aportan la mayor biomasa entre el 40 y $98 \%$ de la densidad total del mesozooplancton en el Océano Pacífico suelen ser dominantes (Pakhomov et al., 2000; Bernard and Froneman, 2002; Cabal et al., 2002; López, 2012; Jaimes and López, 2013; López and Mojica, 2015; Dorado-Roncancio, 2018). The main families in Antarctica are represented by Oithonidae, Oncaeidae, Pseudocalanidae, Calanidae and Metridae (Cabal et al., 2002). Some copepod species have shown responses to photic stimuli and other aspects of basic biology that involve visually ontogenetic behaviors during vertical migrations. These behaviors are driven by copepod eyes, including nauplious eyes and Gicklhorn organs (photoreceptor function found in the genera Calanus and Euchaeta), which vary widely in structure and function between species (Buskey et al., 2012). However, their distribution in water bodies is closely related by local and regional circulation patterns, as well as largescale oceanographic processes such as El Nino Southern Oscillation events, which determine physicochemical and biological characteristics in the water column (López, 2008; Steenbeek et al., 2014).

In the South Pacific, the waters of Panamá, Colombia, Ecuador, Peru and Chile are located with hydrodynamic conditions and changing physicochemical characteristics due to permanent disturbances in these areas, mainly due to upwelling events that occur temporarily, 
En el Pacífico Sur, se localizan aguas de Panamá, Colombia, Ecuador, Perú y Chile con condiciones hidrodinámicas y características fisicoquímicas cambiantes a causa de permanentes perturbaciones en estas áreas, principalmente por los eventos de surgencia que ocurren de manera temporal incrementándose cuando el ímpetu del viento es más fuerte favoreciendo la productividad marina. La dinámica de corrientes representada principalmente por la corriente de Humboldt, la Contracorriente Ecuatorial, la Corriente del Golfo de Panamá y la Corriente de Colombia, se encuentran afectadas por el desplazamiento de la Zona de Convergencia Intertropical- ZCIT y la ocurrencia periódica de eventos El Niño y La Niña condicionan la dinámica climática y afecta la dispersión de todo el plancton (CCCP, 2002; Tejada et al., 2003).

Hacia el norte de Perú la productividad es alta debido a que las surgencias son permanentes todo el año, aunque estas se intensifican durante el invierno cuando la fuerza del viento es más fuerte desplazando masas con gran aporte de nutrientes (FAO, 1995); la surgencia en el centro de Chile tiene menos intensidad y ocurre durante el verano y primavera, cuando se favorece el crecimiento del fitoplancton (CPPS, 2014). Sin embargo hacia el norte de Chile los valores históricos de temperatura y salinidad indican que los afloramientos ocurren durante todo el año favorecidos por la acción de los vientos siendo más fuertes en verano y más débiles durante el invierno (Marín y Olivares, 1999; Blanco et al., 2001; Santander et al, 2001). Esta área está denominada como una de las más productivas para las pesquerías, capturándose peces pelágicos de Chile y Perú, entre 5,6 y 8,0 MMT (Millones de Toneladas Métricas) por año y aproximadamente un tercio son peces pelágicos pequeños como anchoas y sardinas (Blanco et al., 2001).

La ecorregión Antártica e islas del Atlántico Sur se caracteriza por presentar las temperaturas más bajas; hacia el interior de la masa continental Antártica se puede considerar también como la región más desértica del planeta, ya que, a pesar de la gran disponibilidad de hielo, el agua en estado líquido es sumamente escasa (Izaguirre y Sánchez, 2005). Específicamente el Estrecho de Gerlache es un sistema de cuencas semi-cerradas al sur por la Península Antártica y al norte por el Archipiélago Palmer, posee una longitud de 100 a $150 \mathrm{~km}$ y un ancho característico de 40 a $50 \mathrm{~km}$ (Anderson, 1999; Lonin, 2015). Oceanográficamente, en el Estrecho de Gerlache la circulación está controlada por una corriente superficial que se sitúa en la zona increasing when the wind impetus is stronger, and thus favoring marine productivity. The dynamics of currents, mainly the Humboldt Current, the Equatorial Countercurrent, the Gulf of Panama Current and the Colombia Current are affected by the displacement of the Zone of Intertropical Convergence - ZCIT and the periodic occurrence of El Niño and La Niña events condition the climatic dynamics and affect the dispersion of all the plankton (CCCP, 2002; Tejada et al., 2003).

Towards the north of Perú productivity is high due to the fact that the upwellings are permanent all year round, although these intensify during the winter when the wind force is stronger, displacing masses with a great contribution of nutrients (FAO, 1995), whereas the upwelling in central Chile is less intense and occurs during the summer and spring favoring phytoplankton growth (CPPS, 2014). However towards the north of Chile, the historical values of temperature and salinity indicate that the upwellings occur during the whole year favored by the action of the winds which are stronger in summer and weaker during the winter (Marín and Olivares, 1999; Blanco et al., 2001; Santander et $a l, 2001)$. This area is known as one of the most productive for fisheries where pelagic fish are caught from Chile and Peru, between 5.6 and 80 millions of metric tons per year and approximately one third are small pelagic fish such as anchovies and sardines (Blanco et al., 2001).

The ecoregion of Antarctica and the islands of the South Atlantic, characterized by having the lowest temperatures and towards the interior of the Antarctic continental mass, can also be considered the most barren region of the planet, since, in spite of the great availability of ice, water in liquid state is extremely scarce (Izaguirre and Sánchez, 2005). Specifically, the Strait of Gerlache -100 to $150 \mathrm{~km}$ long and 40 to $50 \mathrm{~km}$ wide- is a semi closed basin system, bordered in the south by the Antarctic Peninsula and in the north by the Palmer Archipelago (Anderson, 1999; Lonin, 2015). Oceanographically, in the Strait of Gerlache the circulation is controlled by a surface current that is located in the central zone of the Strait and that moves towards the Strait of Bransfield in a northeastern direction (Bárcena et al., 2005). Surface and deep waters come from the Weddell and Bellingshausen seas. In general, the circulation in the study area is determined by the main Antarctic Circumpolar Current, which varies seasonally (García et al., 2002). 
central del estrecho y que se desplaza hacia el estrecho de Bransfield en dirección noreste (Bárcena et al., 2005). Las aguas superficiales y profundas provienen de los mares de Weddell y Bellingshausen. En general la circulación en el área de estudio está determinada por la corriente principal circumpolar antártica, la cual varía estacionalmente (García et al., 2002).

La dinámica en estas áreas antárticas es constante por el trasporte de Ekman que ocasiona una circulación de aguas superficiales hacia el noreste, alejándose del continente por acción de los vientos. La Corriente Antártica Costera- CCA, que se da por procesos de divergencia, es común que por medio de estos afloramientos llegue a superficie aguas ricas en nutrientes provenientes del Atlántico norte (NADW, por sus siglas en inglés). El mar de Weddell también ayuda a generar la formación de masas de aguas que juegan un papel primordial en la génesis de hielo en las capas superficiales para determinar la dinámica en estas zonas (Loeb et al., 1993; Van der Molen, 2003). También es importante la influencia de otras masas de aguas superficiales que hacen parte de la circulación en el polo sur como es el Agua Subsuperficial Subtropical -SUW, la Corriente Costera- CC y la Confluencia Weddell-Escocia CWE (Loeb et al., 1993, 1997).

Dentro de la comunidad zooplanctónica en la Antártica el grupo que predomina son los copépodos y después se destaca el krill, que está compuesto por diferentes especies de eufáusidos los cuales son alimento tanto de invertebrados como vertebrados marinos y también temporalmente predominan organismos gelatinosos como las salpas (Pascal, 2012). Las comunidades zooplanctónicas del océano Sur pueden estar dominadas por tres grupos taxonómicos: las salpas, el krill o los copépodos (Siegel y Loeb 1995, Hosie, 2000). Aunque el krill es el taxón dominante en las comunidades macrozooplanctónicas en la Antártica ( Laws, 1985; Hopkins, 1985), se ha observado sin embargo que los copépodos pueden dominar tanto en biomasa como en abundancia (Cabal et al., 2002). También existen otras especies de zooplancton como poliquetos, ostrácodos, apendicularios, anfípodos y quetognatos, pero su contribución es menor (Knox, 2006).

Colombia desde 2014 hace parte del Tratado Antártico Internacional, en el cual ha participado en diferentes cruceros de investigación y generado alianzas estratégicas para el desarrollo de la ciencia en este campo. Con el propósito de contribuir al conocimiento del zooplancton e inferir si esta comunidad puede tener un
The dynamics in these Antarctic zones are constant due to the transport of Ekman, which causes a circulation of surface water towards the northeast, moved away from the continent by the winds. The Coastal Antarctic Current - CCA, is given by divergence processes, and it is common for nutrients-rich waters from the North Atlantic NADW to reach the surface through these upwellings. The Weddell Sea also helps to generate the formation of water masses that play a primary role in the formation of ice in the surface layers to determine the dynamics in these areas (Loeb et al., 1993; Van der Molen, 2003). Also important is the influence of other bodies of surface water that are part of the circulation at the South Pole such as Subtropical Subsurface Water -SUW-, Coastal Current - CC-, and the WeddellScotia Confluence -WSC- (Loeb et al., 1993, 1997).

Copepods are the predominant group within the zooplanktonic community in Antarctica, followed by krill, which is made up of different species of euphausiids, which are food for both invertebrates and marine vertebrates. Gelatinous organisms such as salps also predominate temporarily (Pascal, 2012).

Southern Ocean zooplankton communities may be dominated by three taxonomic groups: salps, krill or copepods (Siegel and Loeb 1995; Hosie, 2000). Although krill is the dominant taxon in Antarctic macrozooplanktonic communities (Laws 1985, Hopkins 1985), it has nevertheless been observed that copepods can dominate both in biomass and abundance (Cabal et al., 2002). There are also other species of zooplankton such as polychaetes, ostracods, appendicularies, amphipods and chaetognaths, but their contribution is smaller (Knox, 2006).

Since 2014, Colombia has been part of the International Antarctic Treaty, in which it has participated in different research cruises and generated strategic alliances for the development of science in this field. In order to contribute to the knowledge on zooplankton and infer whether this community can establish some form of connectivity between tropical and polar systems, the study describes the taxonomic composition, distribution and abundance of zooplankton distributed between 0 and $200 \mathrm{~m}$ deep from the Peruvian Pacific to Antarctica.

The null hypothesis states that zooplankton density is lower in Antarctic waters than in the South Pacific, based on the idea that copepods are the dominant community in zooplankton, one would expect to find the copepods of the orders Calanoidea and some Poecilostomatoids, which have been reported to be dominant throughout the South 
tipo de conectividad entre sistemas tropicales y polares se describe la composición taxonómica, distribución y abundancia del zooplancton distribuido entre los 0 y $200 \mathrm{~m}$ de profundidad desde el Pacífico peruano hasta la Antártica.

La hipótesis nula plantea que la densidad zooplanctónica es menor en las aguas Antárticas que en el Pacífico sur, partiendo de la idea que los copépodos son la comunidad que domina en el zooplancton, se esperaría encontrar como dominantes los copépodos de los órdenes Calanoidea y algunos Poecilostomatoida, los cuales han sido descritos a lo largo del Pacífico sur (Monsalve, 1976; López, 2012; Pascal, 2012; Jaimes y López, 2013; López y Mojica, 2015; Medellín-Mora, 2016; Dorado-Roncancio, 2018) sin embargo, existen muchos factores que pueden afectar directa o indirectamente la densidad de estos grupos, ya sea por factores fisicoquímicos y/o ambientales o por la competencia interespecífica dentro del zooplancton con organismos gelatinosos (salpas) y/o eufáusidos que suelen alimentarse de copépodos.

\section{MATERIALES Y MÉTODOS}

Entre diciembre de 2016 y febrero de 2017 se llevó a cabo la III Expedición Científica de Colombia a la Antártica "Almirante Padilla" Verano Austral 2016-2017, la cual, se desarrolló en el buque ARC "20 de Julio". Las estaciones de muestreo abarcaron un gradiente latitudinal desde el Pacífico ecuatorial hasta las costas chilenas (4 estaciones, Figura 1), así como una retícula de muestreo en el Estrecho de Gerlache (10 estaciones, Figura 2) en la Península Antártica.

Para el componente zooplanctónico se realizaron 14 arrastres verticales, desde los 0 a $200 \mathrm{~m}$ de profundidad, utilizando una red de ojo de malla de $250 \mu \mathrm{m}$ y $60 \mathrm{~cm}$ de diámetro de boca. Para estimar el volumen filtrado se acopló a la red un flujómetro Hydro Bios Kiel ${ }^{\circledR}$, previamente calibrado y se aplicó el método estándar propuesto por Boltovskoy (1981), que contempla tiempo de arrastre y revoluciones del flujómetro. Posteriormente, las muestras fueron recolectadas en frascos plásticos de $125 \mathrm{~mL}$, empleando como agente preservante y fijador formaldehido diluido al 8\%, preparado con agua de mar filtrada y tamponado con ácido bórico (APHA et al., 2005). Las muestras fueron llevadas al laboratorio de la Universidad de Bogotá Jorge Tadeo Lozano, sede Santa Marta, donde se adelantó la limpieza de la totalidad de cada muestra. La separación, conteo e identificación de los
Pacific (Monsalve, 1976; López, 2012; Pascal, 2012; Jaimes and López, 2013; López and Mojica, 2015; MedellínMora, 2016; Dorado-Roncancio, 2018). However, there are many factors that can directly or indirectly affect the density of these groups, either by physicochemical and/ or environmental factors or by interspecific competition within zooplankton with gelatinous organisms (salps) and/ or euphausiids that usually feed on copepods.

\section{MATERIALS AND METHODS}

The III Colombian Scientific Expedition to the Antarctic "Almirante Padilla" Austral Summer 2016-2017 took place between December 2016 and February 2017 on the ARC " 20 de Julio" vessel. The sampling stations covered a latitudinal gradient from the equatorial Pacific to the Chilean coasts (4 stations, Figure 1), as well as a sampling grid in the Strait of Gerlache (10 stations, Figure 2 ) in the Antarctic Peninsula.

For the zooplanktonic component, 14 vertical trawls from 0 to $200 \mathrm{~m}$ deep were carried out using a $250 \mu \mathrm{m}$ mesh eye net and $60 \mathrm{~cm}$ mouth diameter. In order to estimate the filtered volume, a previously calibrated Hydro Bios Kiel ${ }^{\circledR}$ flowmeter was attached to the net and the standard method proposed by Boltovskoy (1981), was applied, which includes drag-out time and flowmeter revolutions. Subsequently, the samples were collected in $125 \mathrm{~mL}$ plastic vials, using $8 \%$ diluted formaldehyde as a preserving agent and fixative, prepared with filtered seawater and buffered with boric acid (APHA et al., 2005). The samples were taken to the laboratory at Universidad de Bogotá Jorge Tadeo Lozano, Santa Marta Campus, where they were cleaned. The separation, counting and identification of the zooplankters was taken to the family taxonomic level with the help of specialized literature by Björberg (1981), Boltovskoy (1981), Nishida (1985), Campos-Hernández and Suárez-Morales (1994), Heron \& Bradford- Grieve (1995), Palomares et al. (1998), Bradford-Grieve et al. (1999) and Boxhall and Halsey (2004) and using a 32x LEICA MZ8 stereoscope. The densities of the organisms collected were recorded in ind $\bullet 100 \mathrm{~m}^{-3}$, calculated using the equation:

Density (ind $\left.100 \mathrm{~m}^{-3}\right)=\mathrm{C} \bullet 100 / \mathrm{v}$, where $\mathrm{C}$, is the quantity of organisms obtained in the count and $\mathrm{v}$, corresponds to the volumes filtered in each trawl.

In order to process the information, data matrices were initially constructed, relating the number of organisms 
zooplánctones se llevó hasta el nivel taxonómico de familia con la ayuda de literatura especializada de Björberg (1981), Boltovskoy (1981), Nishida (1985), Campos-Hernández y Suárez-Morales (1994), Heron y Bradford- Grieve (1995), Palomares et al. (1998), Bradford- Grieve et al. (1999) y Boxhall y Halsey (2004), haciendo uso de un estereoscopio LEICA MZ8 de 32x. Las densidades de los organismos recolectados se registraron en ind $\bullet 100 \mathrm{~m}^{-3}$, las cuales se calcularon a partir de la ecuación:

Densidad (ind $\left.\bullet 100 \mathrm{~m}^{-3}\right)=\mathrm{C} \bullet 100 / \mathrm{v}$, donde $\mathrm{C}$, es la cantidad de organismos obtenidos en el conteo y v, corresponde a los volúmenes filtrados en cada arrastre. by families/morphotypes identified in each of the stations. Pie charts were drawn up using this information. The community was described using Shannon-Wiener diversity indices $\left(\mathrm{H}^{\prime}\right)$, Margalef's richness index:(d) and Pielou uniformity or evenness $\left(\mathrm{J}^{\prime}\right)$ (Moreno, 2001). To determine whether there were differences in ecological attributes between South Pacific stations and Antarctic stations, univariate analyses were conducted, such as t-student tests (T) (Zar, 2010). The previous test was defined according to the fulfillment of the assumptions of normality and homogeneity of variances using the STATGRAPHICS Centurion $®$ statistical program.

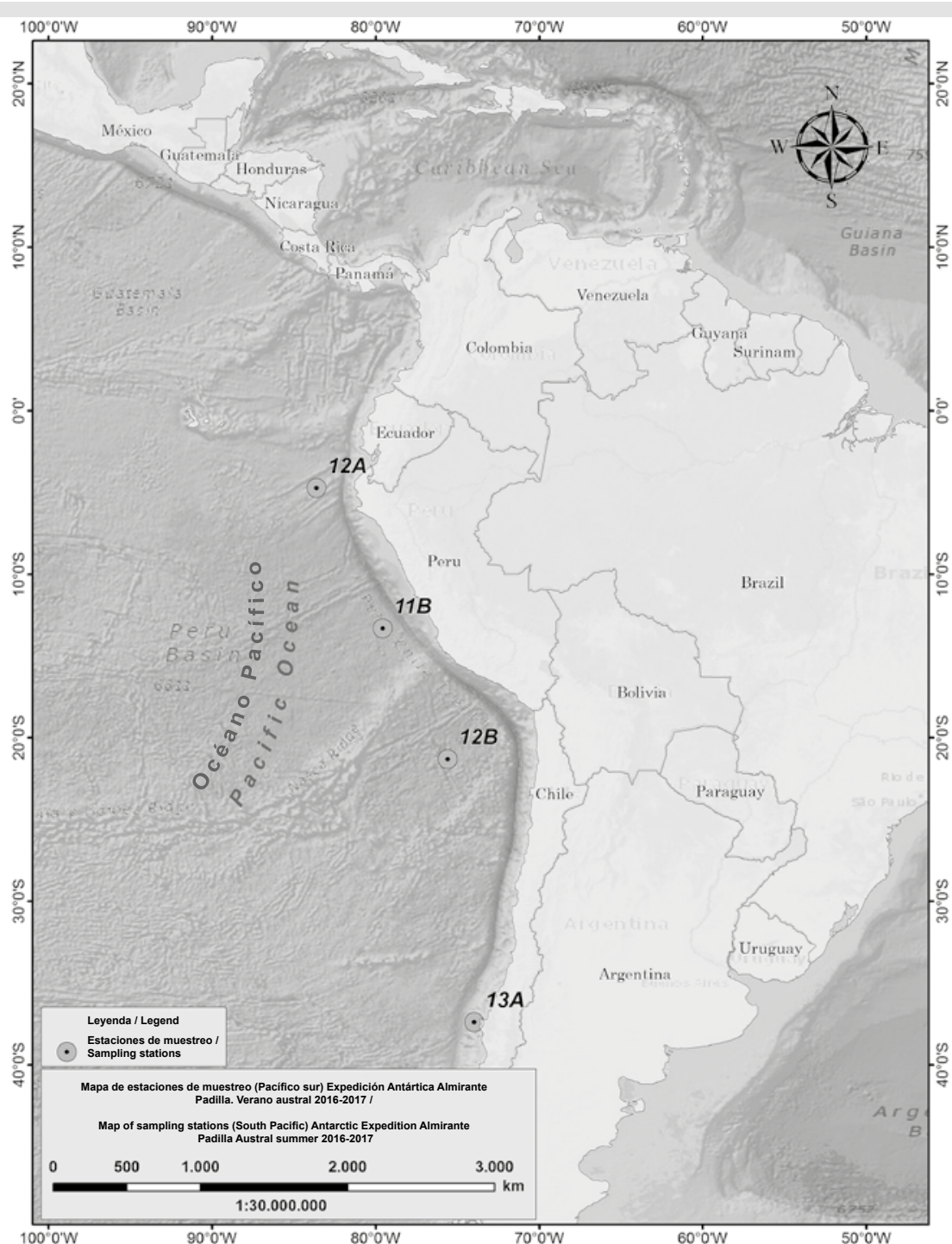

Figura 1. Mapa de las Estaciones muestreadas en el gradiente latitudinal a lo largo del Pacífico Sur, Expedición Antártica Almirante Padilla, Verano Austral 2016-2017.

Figure 1. Map of Stations sampled in the latitudinal gradient along the South Pacific, Almirante Padilla Antarctic Expedition, Austral Summer 2016-2017. 


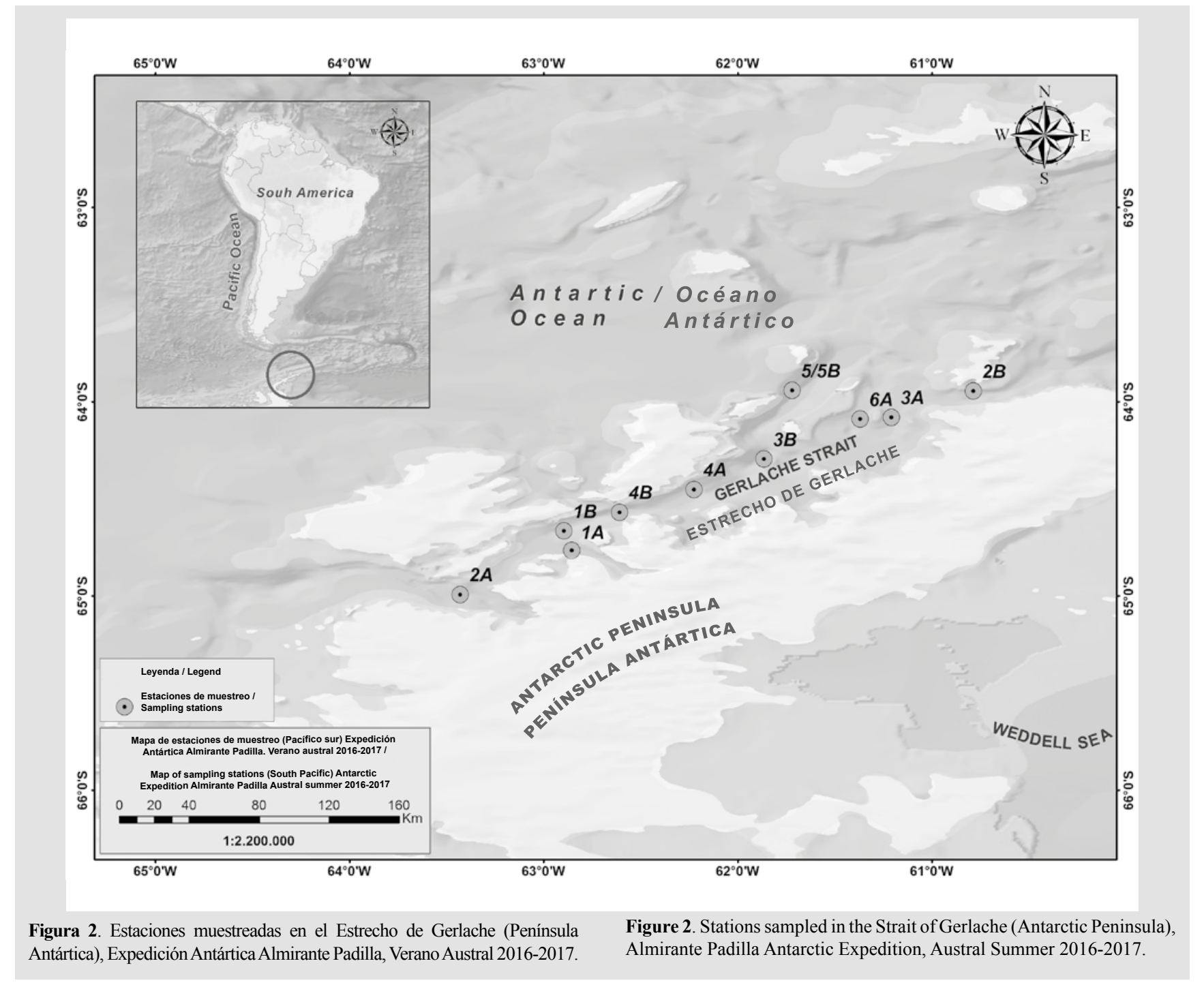

Para el tratamiento de la información se construyeron inicialmente matrices de datos, relacionando el número de organismos por las familias/morfotipos identificados en cada una de las estaciones. Con esta información, se hicieron gráficos de composición tipo pastel. La comunidad se describió a partir de índices de diversidad de Shannon-Wiener $\left(\mathrm{H}^{\prime}\right)$, riqueza de Margalef (d) y uniformidad o equitatividad de Pielou ( $\mathrm{J}^{\prime}$ ) (Moreno, 2001). Para determinar si existían diferencias de los atributos ecológicos entre estaciones del Pacífico Sur con estaciones de la Antártica, se realizaron análisis univariados, como las pruebas $t$-student (T) (prueba paramétrica) (Zar, 2010). La anterior prueba se definió de acuerdo al cumplimiento de los supuestos de normalidad y homogeneidad de varianzas utilizando el programa estadístico STATGRAPHICS Centurion®.
Multivariate techniques were used to evaluate and identify possible spatial patterns (stations) in the zooplankton community structure. For this purpose, the transformation of data from fourth-root was carried out according to the model proposed by Taylor (1961). Based on these values, a classification analysis was performed based on the unweighted average ligation grouping technique (UPGMA) (Field et al., 1982; Ramírez, 1999), using the Primer $6+$ Permanova ${ }^{\circledR}$ program. This analysis allows us to observe associations in terms of composition and abundance, according to the degree of similarity estimated with the Bray-Curtis index. Additionally, the confenetic index was established with the Pearson correlation $(\alpha=0.05)$ with a $95 \%$ confidence between each pair of variables. 
Se emplearon técnicas multivariadas con la finalidad de evaluar e identificar los posibles patrones espaciales (estaciones) en la estructura de la comunidad zooplanctónica. Para esto, previamente se realizó la transformación de raíz cuarta a los datos, según el modelo propuesto por Taylor (1961). A partir de estos valores, se adelantó un análisis de clasificación basado en la técnica de agrupamiento de ligamiento promedio no ponderado (UPGMA) (Field et al., 1982; Ramírez, 1999), utilizando el programa Primer $6+$ Permanova ${ }^{\circledR}$. Este análisis permite observar asociaciones en términos de composición y abundancia, de acuerdo con el grado de similitud estimado con el índice de Bray-Curtis. Adicionalmente, el índice confenético se estableció con la correlación de Pearson $(\alpha=$ $0,05)$ con una confianza de $95 \%$ entre cada par de variables.

\section{RESULTADOS}

La comunidad zooplanctónica estuvo conformada por un total de 75 familias/morfotipos $(\mathrm{f} / \mathrm{m})$, pertenecientes a 32 órdenes. El orden Calanoida (20\%) y los Perciformes (12\%) predominaron en este estudio. Los estadios tempranos como huevos y larvas de peces, bivalvos, cipris, entre otros, aportaron 54\% de importancia relativa (Figura 3). Dentro de los copépodos, las familias Calanidae, Paracalanidae, Clausocalanidae, Sagittidae, Oncaeidae y Eucalanidae fueron en términos de abundancia las más representativas del muestreo. Cabe resaltar que para las estaciones $4 \mathrm{~A}, \mathrm{AB}$ y 6A, muestreadas en el Estrecho de Gerlache se encontraron densidades altas de salpas, más específicamente del grupo de las Thaliacea con hasta 281,62 ind $\bullet 100 \mathrm{~m}^{-3}$ para la estación 4B (Tabla 1). Igualmente, se registra la aparición de salpas en una estación del Pacífico Sur (11B) con 278,75 ind•100 $\mathrm{m}^{-3}$. En este estudio se encontraron otros organismos gelatinosos como quetognatos de la familia Saggitidae (salpas), que presentaron la más alta densidad (2508,78 ind $\bullet 100 \mathrm{~m}^{-3}$ ), para la estación 4B. Esta familia se registra en densidades menores para las otras estaciones tanto en la Antártica como en el Pacífico Sur. Otro grupo importante en este estudio es el krill, representado principalmente por la familia Euphausiidae que, como era de esperarse, se encontró en mayor densidad en la estación 3B (Antártica) con $\left(2055,90\right.$ ind $\left.\bullet 100 \mathrm{~m}^{-3}\right)$.El krill constituye el alimento predilecto de ballenas y otros mamíferos marinos que viven en la Antártica, aunque también se encontró en menores concentraciones en las otras estaciones muestreadas en este estudio (Tabla 1; Figure 3).

\section{RESULTS}

The zooplankton community consisted of a total of 75 families/morphotypes ( $\mathrm{f} / \mathrm{m})$, belonging to 32 orders. The order Calanoid (20\%) and Perciformes (12\%) predominated in this study. Early stages such as fish eggs and larvae, bivalves, and cypris, among others, contributed $54 \%$ of relative importance (Figure 3). Within the copepods, the families Calanidae, Paracalanidae, Clausocalanidae, Sagittidae, Oncaeidae and Eucalanidae were the most representative of the sampling in terms of abundance. It should be noted that for the stations ( $4 \mathrm{~A}, \mathrm{AB}$ and $6 \mathrm{~A}$ ) sampled in the Strait of Gerlache high densities of salps were found, more specifically from the Thaliacea group with up to 281.62 ind $\bullet 100 \mathrm{~m}^{-3}$ for station 4B (Table 1). Likewise, the appearance of salps is reported in a South Pacific station (11B) with 278.75 ind $\bullet 100 \mathrm{~m}^{-3}$. In this study, other gelatinous organisms were found, such as chaetognaths of the Saggitidae family (salps), which presented the highest density $\left(2508.78\right.$ ind $\left.\bullet 100 \mathrm{~m}^{-3}\right)$, for season 4B. This family is reported in lower densities for the other stations in both Antarctica and the South Pacific. Another important group in this study is krill, represented mainly by the Euphausiidae family, which, as expected, was found in a higher density in station 3B (Antarctica) with (2055.90 ind $100 \mathrm{~m}^{-3}$ ). Krill is the favorite food of whales and other marine mammals living in Antarctica, although it was also found in lower concentrations in the other stations sampled in this study (Table 1; Figure 3).

The mean and standard deviation of the number of families/morphotypes per sampled station was $22.64 \pm$ 11.91 , with values ranging from $10-47 \mathrm{f} / \mathrm{m}$ in station $2 B$ and $12 \mathrm{~A}$, respectively, the first corresponding to the Antarctic and the last station to the Equatorial Pacific. In the grid showing the stations, it is important to highlight that, although some families/morphotypes are reported, both in the Pacific and in Antarctica, there was always less richness in the Strait of Gerlache. The average density was 9483 ind $100 \mathrm{~m}^{-3}$ with a range of data between 1044 and 37834 ind $100 \mathrm{~m}^{-3}$, the greatest abundances being found at stations 11B (37834 ind $\bullet 100 \mathrm{~m}^{-3}$ ) and 12A (32444 ind $100 \mathrm{~m}^{-3}$; Table 1). Among the most important groups, the Calanidae family presented the highest density in the entire latitudinal gradient, 6057 ind $100 \mathrm{~m}^{-3}$ in station 11B (Pacific), and 1594 ind $\bullet 100 \mathrm{~m}^{-3}$ in station 1A (Antarctic) (Table 1). The values of richness, diversity and uniformity showed similar values for all 


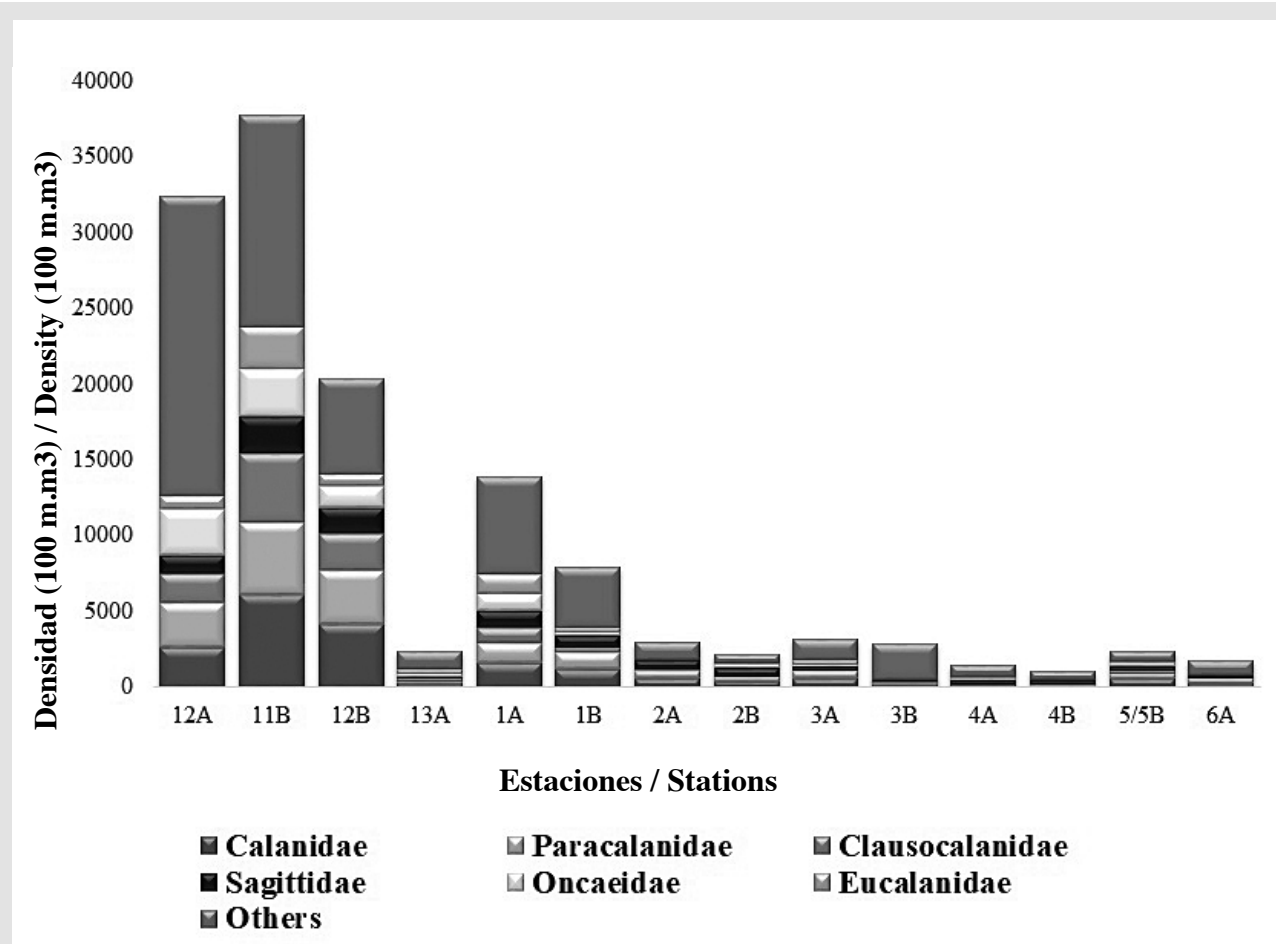

Figura 3. Densidad total y aportes de las familias/morfotipos, identificados en las estaciones muestreadas durante la III Expedición Científica de Colombia a la Antártica "Almirante Padilla" Verano Austral 2016-2017.
Figure 3. Total density and contributions of families/morphotypes, identified in the stations sampled during the III Colombian Scientific Expedition to the Antarctic “Almirante Padilla” Austral Summer 2016-2017
Tabla 1. Densidades de la comunidad zooplanctónica desde el Pacífico Sur hasta la Antártica, parte superior estaciones 12A, 11B, 12B y 13A, correspondientes a las estaciones muestreadas en el Pacífico Sur. Parte inferior, resto de estaciones muestreadas en el Estrecho de Gerlache. Muestra las densidades de las Familias-Morfotipos expresadas en ind. $\bullet 100 \mathrm{~m}^{-3}$.
Table 1. Density of the zooplankton community from the South Pacific to Antarctica, upper part stations 12A, 11B, 12B and 13A, corresponding to the sampled stations in the South Pacific. Lower part, remaining stations sampled in the Strait of Gerlache. It shows the densities of the FamiliesMorphotypes expressed in ind. $\bullet 100 \mathrm{~m}^{-3}$.

\begin{tabular}{|c|c|c|c|c|}
\hline $\begin{array}{l}\text { Familias-Morfotipos / } \\
\text { Estaciones } \\
\text { Families-Morphotypes / } \\
\text { Stations }\end{array}$ & $12 \mathrm{~A}$ & $11 B$ & $12 B$ & $13 \mathrm{~A}$ \\
\hline Abylidae & 101.12 & 0.00 & 0.00 & 0.00 \\
\hline Acartiidae & 0.00 & 1258.61 & 0.00 & 0.00 \\
\hline Alciopidae & 379.21 & 523.72 & 177.98 & 12.54 \\
\hline Alpheidae & 0.00 & 0.00 & 0.00 & 17.11 \\
\hline Atherinopsidae & 0.00 & 67.58 & 0.00 & 0.00 \\
\hline Atlantidae & 227.53 & 0.00 & 0.00 & 0.00 \\
\hline Augaptillidae & 438.20 & 0.00 & 487.80 & 99.21 \\
\hline Branchiostomatidae & 210.67 & 126.71 & 0.00 & 0.00 \\
\hline Calanidae & 2578.64 & 6056.55 & 4093.59 & 239.48 \\
\hline Candaciidae & 210.67 & 878.49 & 316.41 & 0.00 \\
\hline Carangidae & 0.00 & 42.24 & 52.74 & 0.00 \\
\hline
\end{tabular}




\begin{tabular}{|c|c|c|c|c|}
\hline $\begin{array}{c}\text { Familias-Morfotipos / } \\
\text { Estaciones } \\
\text { Families-Morphotypes / } \\
\text { Stations }\end{array}$ & $12 \mathrm{~A}$ & $11 B$ & $12 B$ & $13 \mathrm{~A}$ \\
\hline Cavoliniidae & 134.83 & 135.15 & 0.00 & 0.00 \\
\hline Centropagidae & 758.42 & 726.45 & 217.53 & 70.70 \\
\hline Cyprinidae & 0.00 & 160.49 & 0.00 & 57.02 \\
\hline Clausocalanidae & 1988.76 & 4502.29 & 2406.06 & 167.63 \\
\hline Corycaeidae & 2957.85 & 2382.07 & 1219.51 & 0.00 \\
\hline Dyphidae & 0.00 & 253.41 & 0.00 & 11.40 \\
\hline Engraulidae & 25.28 & 59.13 & 13.18 & 0.00 \\
\hline Eucalanidae & 867.97 & 2846.66 & 731.71 & 248.60 \\
\hline Euchaetidae & 0.00 & 777.13 & 0.00 & 0.00 \\
\hline Euphausiidae & 522.47 & 33.79 & 349.37 & 52.46 \\
\hline Evermannellidae & 0.00 & 50.68 & 0.00 & 0.00 \\
\hline Gempylidae & 4980.32 & 25.34 & 0.00 & 0.00 \\
\hline Geryonidae & 0.00 & 295.65 & 0.00 & 0.00 \\
\hline Globigerinidae & 733.14 & 0.00 & 0.00 & 0.00 \\
\hline Gobiidae & 0.00 & 25.34 & 0.00 & 0.00 \\
\hline Gonostomatidae & 0.00 & 67.58 & 6.59 & 0.00 \\
\hline Halocyprinidae & 598.31 & 295.65 & 342.78 & 142.55 \\
\hline Huevo pez / Fish egg & 514.04 & 143.60 & 46.14 & 0.00 \\
\hline Hyperiidae & 294.94 & 270.31 & 164.80 & 0,00 \\
\hline Isopodo & 33.71 & 0.00 & 0.00 & 18.25 \\
\hline Labridae & 0.00 & 50.68 & 0.00 & 0.00 \\
\hline $\begin{array}{l}\text { Larva cirripedio / } \\
\text { Cirripedia larva }\end{array}$ & 0.00 & 0.00 & 0.00 & 28.51 \\
\hline Cephalopod larva & 134.83 & 59.13 & 0.00 & 0.00 \\
\hline Decapod larva & 0.00 & 0.00 & 0.00 & 25.09 \\
\hline Mollusk larva & 117.98 & 0.00 & 0.00 & 0.00 \\
\hline Fish larva & 185.39 & 160.49 & 19.78 & 0.00 \\
\hline Zoea larva & 193.82 & 0.00 & 105.47 & 0.00 \\
\hline Lestrigonidae & 50.56 & 0.00 & 0.00 & 0.00 \\
\hline Limacinidae & 143.26 & 0.00 & 98.88 & 0.00 \\
\hline Linophrynidae & 0.00 & 33.79 & 0.00 & 0.00 \\
\hline Lucicutidae & 438.20 & 0.00 & 527.36 & 88.95 \\
\hline Majidae & 0.00 & 0.00 & 0.00 & 3.42 \\
\hline Membraniporidae & 0.00 & 0.00 & 0.00 & 58.16 \\
\hline Miraciidae & 67.42 & 0.00 & 0.00 & 0.00 \\
\hline Metridinidae & 42.13 & 0.00 & 0.00 & 152.81 \\
\hline
\end{tabular}




\begin{tabular}{|c|c|c|c|c|}
\hline $\begin{array}{l}\text { Familias-Morfotipos / } \\
\text { Estaciones } \\
\text { Families-Morphotypes / } \\
\text { Stations }\end{array}$ & $12 \mathrm{~A}$ & $11 B$ & $12 B$ & $13 \mathrm{~A}$ \\
\hline Myctophidae & 0.00 & 92.92 & 0.00 & 0.00 \\
\hline Mysidae & 0.00 & 354.78 & 0.00 & 0.00 \\
\hline Nomeidae & 8.43 & 33.79 & 0.00 & 0.00 \\
\hline Oikopleuridae & 185.39 & 0.00 & 0.00 & 15.97 \\
\hline Oithonidae & 303.37 & 0.00 & 435.07 & 71.84 \\
\hline Oncaeidae & 3008.42 & 3057.84 & 1509.55 & 230.35 \\
\hline Paracalanidae & 2915.72 & 4721.91 & 3566.24 & 167.63 \\
\hline Paralepididae & 92.70 & 295.65 & 0.00 & 0.00 \\
\hline phaenidae & 25.28 & 0.00 & 0.00 & 0.00 \\
\hline Phosichthyidae & 320.22 & 211.18 & 0.00 & 0.00 \\
\hline Pontellidae & 269.66 & 473.04 & 0.00 & 0.00 \\
\hline Sagittidae & 1196.62 & 2508.78 & 1674.35 & 103.77 \\
\hline Salpidae & 0.00 & 768.68 & 85.70 & 20.53 \\
\hline Sapphirinidae & 278.09 & 650.42 & 65.92 & 34.21 \\
\hline Scaridae & 0.00 & 25.34 & 0.00 & 0.00 \\
\hline Scolectrichidae & 977.52 & 886.94 & 1100.85 & 125.44 \\
\hline Sergestidae & 421.35 & 0.00 & 369.15 & 0.00 \\
\hline Sipunculidae & 109.55 & 0.00 & 0.00 & 10.26 \\
\hline Sphyraenidae & 0.00 & 25.34 & 6.59 & 0.00 \\
\hline Spionidae & 320.22 & 0.00 & 158.21 & 12.54 \\
\hline Squillidae & 25.28 & 0.00 & 0.00 & 0.00 \\
\hline Synodontidae & 0.00 & 76.02 & 0.00 & 0.00 \\
\hline Temoridae & 2014.04 & 1047.44 & 0.00 & 59.30 \\
\hline Tubularidae & 42.13 & 0.00 & 0.00 & 0.00 \\
\hline Gelatinous Thaliacean & 0.00 & 278.75 & 0.00 & 0.00 \\
\hline Copepod & 0.00 & 16.89 & 0.00 & 1.14 \\
\hline Total & 32443.70 & 37834.40 & 20349.31 & 2346.88 \\
\hline Promedio / Average & 450.61 & 525.48 & 282.63 & 32.60 \\
\hline $\begin{array}{l}\text { Desviación estándar / } \\
\text { Standard deviation }\end{array}$ & 903.02 & 1165.65 & 742.86 & 61.37 \\
\hline Mínimo / Minimum & 0.00 & 0.00 & 0.00 & 0.00 \\
\hline Máximo / Maximum & 4980.32 & 6056.55 & 4093.59 & 248.60 \\
\hline
\end{tabular}




\begin{tabular}{|c|c|c|c|c|c|c|c|c|c|c|}
\hline $\begin{array}{l}\text { FAMILIA } \\
\text { FAMILY }\end{array}$ & $\mathbf{1 A}$ & $1 \mathrm{~B}$ & $\mathbf{2 A}$ & $2 \mathrm{~B}$ & 3A & 3B & $4 \mathrm{~A}$ & 4B & $5 / 5 B$ & $6 \mathrm{~A}$ \\
\hline Abylidae & 0.00 & 0.00 & 0.00 & 0.00 & 40.69 & 0.00 & 0.00 & 0.00 & 0.00 & 0.00 \\
\hline Acartiidae & 0.00 & 0.00 & 0.00 & 0.00 & 0.00 & 37.30 & 0.00 & 0.00 & 0.00 & 0.00 \\
\hline Alciopidae & 0.00 & 0.00 & 81.48 & 45.01 & 0.00 & 0.00 & 0.00 & 0.00 & 40.94 & 33.03 \\
\hline Alpheidae & 194.07 & 0.00 & 0.00 & 0.00 & 0.00 & 0.00 & 0.00 & 0.00 & 0.00 & 0.00 \\
\hline Augaptillidae & 762.43 & 624.20 & 0.00 & 135.04 & 664.63 & 116.06 & 149.16 & 27.48 & 106.45 & 132.10 \\
\hline Branchiostomatidae & 0.00 & 771.07 & 183.32 & 0.00 & 0.00 & 0.00 & 0.00 & 0.00 & 0.00 & 0.00 \\
\hline Calanidae & 1594.17 & 1174.96 & 590.70 & 345.10 & 542.55 & 186.52 & 175.80 & 68.69 & 515.85 & 255.94 \\
\hline Candaciidae & 166.35 & 0.00 & 0.00 & 0.00 & 0.00 & 0.00 & 58.60 & 0.00 & 0.00 & 0.00 \\
\hline Carangidae & 0.00 & 0.00 & 61.11 & 0.00 & 0.00 & 0.00 & 0.00 & 0.00 & 0.00 & 0.00 \\
\hline Cyprinidae & 568.35 & 238.66 & 81.48 & 0.00 & 0.00 & 0.00 & 0.00 & 0.00 & 0.00 & 0.00 \\
\hline Clausocalanidae & 942.64 & 348.82 & 0.00 & 0.00 & 0.00 & 132.64 & 0.00 & 0.00 & 180.14 & 0.00 \\
\hline Dyphidae & 0.00 & 0.00 & 81.48 & 0.00 & 27.13 & 0.00 & 0.00 & 0.00 & 0.00 & 41.28 \\
\hline Eucalanidae & 1303.06 & 293.74 & 0.00 & 165.05 & 230.58 & 37.30 & 90.56 & 0.00 & 114.63 & 33.03 \\
\hline Euchaetidae & 0.00 & 0.00 & 40.74 & 0.00 & 0.00 & 0.00 & 0.00 & 0.00 & 0.00 & 0.00 \\
\hline Euphausiidae & 0.00 & 110.15 & 0.00 & 0.00 & 94.95 & 2055.90 & 42.62 & 0.00 & 0.00 & 33.03 \\
\hline Evermannellidae & 0.00 & 0.00 & 122.21 & 0.00 & 0.00 & 0.00 & 0.00 & 0.00 & 0.00 & 0.00 \\
\hline Gobiidae & 0.00 & 0.00 & 61.11 & 0.00 & 0.00 & 0.00 & 0.00 & 0.00 & 0.00 & 0.00 \\
\hline Halocyprinidae & 790.15 & 0.00 & 0.00 & 120.04 & 176.33 & 58.03 & 63.93 & 34.34 & 131.01 & 255.94 \\
\hline Harpagiferidae & 0.00 & 0.00 & 224.06 & 0.00 & 27.13 & 0.00 & 0.00 & 0.00 & 0.00 & 0.00 \\
\hline Huevo Pez / Fish egg & 402.01 & 91.79 & 0.00 & 0.00 & 54.26 & 0.00 & 0.00 & 27.48 & 24.56 & 57.79 \\
\hline Hyperiidae & 0.00 & 0.00 & 40.74 & 0.00 & 0.00 & 0.00 & 0.00 & 13.74 & 0.00 & 0.00 \\
\hline Isopodo & 0.00 & 0.00 & 0.00 & 0.00 & 0.00 & 0.00 & 0.00 & 27.48 & 40.94 & 33.03 \\
\hline Labridae & 0.00 & 0.00 & 40.74 & 0.00 & 0.00 & 0.00 & 0.00 & 0.00 & 0.00 & 0.00 \\
\hline $\begin{array}{l}\text { Larva Cirripedio / } \\
\text { Cirripedia larva }\end{array}$ & 152.49 & 0.00 & 0.00 & 0.00 & 0.00 & 0.00 & 0.00 & 0.00 & 40.94 & 0.00 \\
\hline Decapod larva & 83.17 & 0.00 & 0.00 & 0.00 & 0.00 & 0.00 & 31.96 & 13.74 & 57.32 & 0.00 \\
\hline Mollusk larva & 0.00 & 0.00 & 0.00 & 0.00 & 0.00 & 0.00 & 10.65 & 0.00 & 0.00 & 0.00 \\
\hline Fish larva & 13.86 & 0.00 & 0.00 & 0.00 & 40.69 & 0.00 & 0.00 & 0.00 & 0.00 & 0.00 \\
\hline Zoea larva & 41.59 & 0.00 & 0.00 & 0.00 & 0.00 & 0.00 & 0.00 & 0.00 & 0.00 & 0.00 \\
\hline Lucicutidae & 706.98 & 1872.59 & 0.00 & 240.07 & 162.77 & 82.90 & 149.16 & 13.74 & 0.00 & 214.66 \\
\hline Majidae & 0.00 & 0.00 & 183.32 & 0.00 & 0.00 & 0.00 & 0.00 & 0.00 & 0.00 & 0.00 \\
\hline Membraniporidae & 429.73 & 0.00 & 0.00 & 0.00 & 0.00 & 0.00 & 0.00 & 0.00 & 57.32 & 0.00 \\
\hline Miraciidae & 0.00 & 0.00 & 0.00 & 0.00 & 0.00 & 0.00 & 0.00 & 0.00 & 0.00 & 0.00 \\
\hline Metridinidae & 235.66 & 91.79 & 0.00 & 0.00 & 0.00 & 0.00 & 0.00 & 0.00 & 0.00 & 0.00 \\
\hline Mysidae & 0.00 & 0.00 & 0.00 & 0.00 & 0.00 & 0.00 & 0.00 & 13.74 & 0.00 & 0.00 \\
\hline Nototheniidae & 0.00 & 0.00 & 0.00 & 0.00 & 13.56 & 0.00 & 0.00 & 0.00 & 0.00 & 0.00 \\
\hline Oikopleuridae & 152.49 & 0.00 & 0.00 & 0.00 & 0.00 & 0.00 & 0.00 & 0.00 & 73.69 & 0.00 \\
\hline
\end{tabular}




\begin{tabular}{|c|c|c|c|c|c|c|c|c|c|c|}
\hline $\begin{array}{l}\text { FAMILIA } \\
\text { FAMILY }\end{array}$ & $1 \mathrm{~A}$ & $1 \mathrm{~B}$ & $\mathbf{2 A}$ & $2 \mathrm{~B}$ & $\mathbf{3 A}$ & 3B & $4 \mathrm{~A}$ & $4 B$ & $5 / 5 B$ & $6 \mathrm{~A}$ \\
\hline Oithonidae & 249.52 & 0.00 & 0.00 & 0.00 & 94.95 & 20.72 & 0.00 & 34.34 & 0.00 & 66.05 \\
\hline Oncaeidae & 1122.85 & 220.31 & 0.00 & 165.05 & 94.95 & 16.58 & 117.20 & 0.00 & 188.33 & 0.00 \\
\hline Paracalanidae & 1330.78 & 1064.81 & 0.00 & 285.09 & 569.68 & 82.90 & 0.00 & 61.82 & 343.90 & 255.94 \\
\hline Paralepididae & 0.00 & 0.00 & 81.48 & 0.00 & 0.00 & 0.00 & 0.00 & 0.00 & 0.00 & 0.00 \\
\hline Peltidiidae & 0.00 & 0.00 & 488.85 & 0.00 & 0.00 & 0.00 & 0.00 & 34.34 & 0.00 & 0.00 \\
\hline Sagittidae & 1122.85 & 826.14 & 0.00 & 630.19 & 298.40 & 24.87 & 277.02 & 288.49 & 343.90 & 264.20 \\
\hline Salpidae & 0.00 & 0.00 & 0.00 & 0.00 & 27.13 & 0.00 & 0.00 & 13.74 & 0.00 & 0.00 \\
\hline Sapphirinidae & 180.21 & 0.00 & 570.33 & 0.00 & 0.00 & 0.00 & 0.00 & 0.00 & 32.75 & 0.00 \\
\hline Scolectrichidae & 1122.85 & 110.15 & 0.00 & 0.00 & 0.00 & 0.00 & 85.24 & 48.08 & 0.00 & 0.00 \\
\hline Sergestidae & 83.17 & 0.00 & 0.00 & 0.00 & 0.00 & 0.00 & 0.00 & 0.00 & 0.00 & 0.00 \\
\hline Sipunculidae & 110.90 & 128.51 & 0.00 & 45.01 & 40.69 & 0.00 & 21.31 & 27.48 & 24.56 & 74.31 \\
\hline Spionidae & 0.00 & 0.00 & 40.74 & 0.00 & 0.00 & 0.00 & 0.00 & 13.74 & 0.00 & 0.00 \\
\hline Synodontidae & 0.00 & 0.00 & 40.74 & 0.00 & 0.00 & 0.00 & 0.00 & 0.00 & 0.00 & 0.00 \\
\hline Temoridae & 0.00 & 0.00 & 0.00 & 0.00 & 0.00 & 0.00 & 0.00 & 0.00 & 57.32 & 0.00 \\
\hline $\begin{array}{l}\text { Gelatinous } \\
\text { Thaliacean }\end{array}$ & 0.00 & 0.00 & 0.00 & 0.00 & 0.00 & 0.00 & 175.80 & 281.62 & 0.00 & 41.28 \\
\hline Copepod & 55.45 & 0.00 & 0.00 & 0.00 & 0.00 & 0.00 & 0.00 & 0.00 & 0.00 & 0.00 \\
\hline Total & 13917.75 & 7967.70 & 3014.60 & 2175.66 & 3201.05 & 2851.73 & 1449.01 & 1044.05 & 2374.55 & 1791.61 \\
\hline Promedio / Average & 267.65 & 153.23 & 57.97 & 41.84 & 61.56 & 54.84 & 27.87 & 20.08 & 45.66 & 34.45 \\
\hline $\begin{array}{l}\text { Desviación estándar } \\
\text { Standard deviation }\end{array}$ & 434.41 & 366.16 & 133.80 & 113.35 & 146.91 & 285.51 & 59.87 & 56.03 & 101.59 & 75.29 \\
\hline Mínimo / Minimum & 0.00 & 0.00 & 0.00 & 0.00 & 0.00 & 0.00 & 0.00 & 0.00 & 0.00 & 0.00 \\
\hline Máximo / Maximum & 1594.17 & 1872.59 & 590.70 & 630.19 & 664.63 & 2055.90 & 277.02 & 288.49 & 515.85 & 264.20 \\
\hline
\end{tabular}

El promedio y desviación estándar del número de familias/morfotipos por estación muestreada fue de 22,64 \pm 11,91 , oscilando sus valores entre 10- $47 \mathrm{f} / \mathrm{m}$, en la estación $2 \mathrm{~B}$ y $12 \mathrm{~A}$, respectivamente correspondiendo la primera al continente blanco y la última estación al Pacífico ecuatorial. En la retícula de estaciones cabe resaltar que, aunque se registran algunas familias/morfotipos tanto en el Pacífico como en la Antártica, se encontró siempre menos riqueza en el Estrecho de Gerlache. El promedio de densidad fue de 9483 ind $100 \mathrm{~m}^{-3}$ con un ámbito de datos entre $1044 \mathrm{y}$ 37834 ind $100 \mathrm{~m}^{3}$, encontrándose las mayores abundancias en las estaciones 11B (37834 ind $\left.100 \mathrm{~m}^{3}\right)$ y $12 \mathrm{~A}(32444$ ind $100 \mathrm{~m}^{3}$; Tabla 1). Dentro de los grupos más importantes, la familia Calanidae presentó la mayor densidad en todo el gradiente latitudinal, 6057 ind $100 \mathrm{~m}^{-3}$ en la estación 11B (Pacífico), y 1594 ind $\bullet 100 \mathrm{~m}^{-3}$ en la estación 1A (Antártica) stations (Figure 5); uniformity in general terms is high, with values above 0.8 (a maximum of 0.9 in station 4A), with exception of station $3 \mathrm{~B}$ where this index reaches a value of 0.5 . The diversity index ranged from 3.074 to 1.193 , and richness is shown to be highest in Pacific stations with the maximum level in station $12 \mathrm{~A}$ at 4.4 while the minimum was recorded in a South Pole station (2.5 in station 2B).

Four groupings were observed in the classification analysis (Figure 6). Group 1 is represented only by station 2A (Antarctica), which presents the spatial characteristic of being further south of the other sampling points in Antarctica (Figure 2), in addition to presenting low organism density (3015 ind $\left.\bullet 100 \mathrm{~m}^{-3}\right)$, where the greatest abundance was represented by the Calanidae family with 591 ind $\bullet 100 \mathrm{~m}^{-3}$. Group 2 is represented mainly by the rest of Antarctic stations, and the southernmost station 
(Tabla 1). Los valores de riqueza, diversidad y uniformidad presentaron valores similares entre todas las estaciones (Figure 4); la uniformidad en términos generales es alta, con valores por encima de 0,8 (un máximo de 0,9 en la estación 4A), con excepción de la estación 3B en la que este índice alcanza un valor de 0,5. El índice de diversidad osciló entre in the Pacific (13A) with a similarity of between 50 and $70 \%$. Group 3 represents a similarity of approximately $60 \%$, and comprises two stations located in the Strait of Gerlache. These two stations are related due to their low density. Group 4 reaches a similarity of $60 \%$ and includes the remaining stations of the South Pacific, characterized

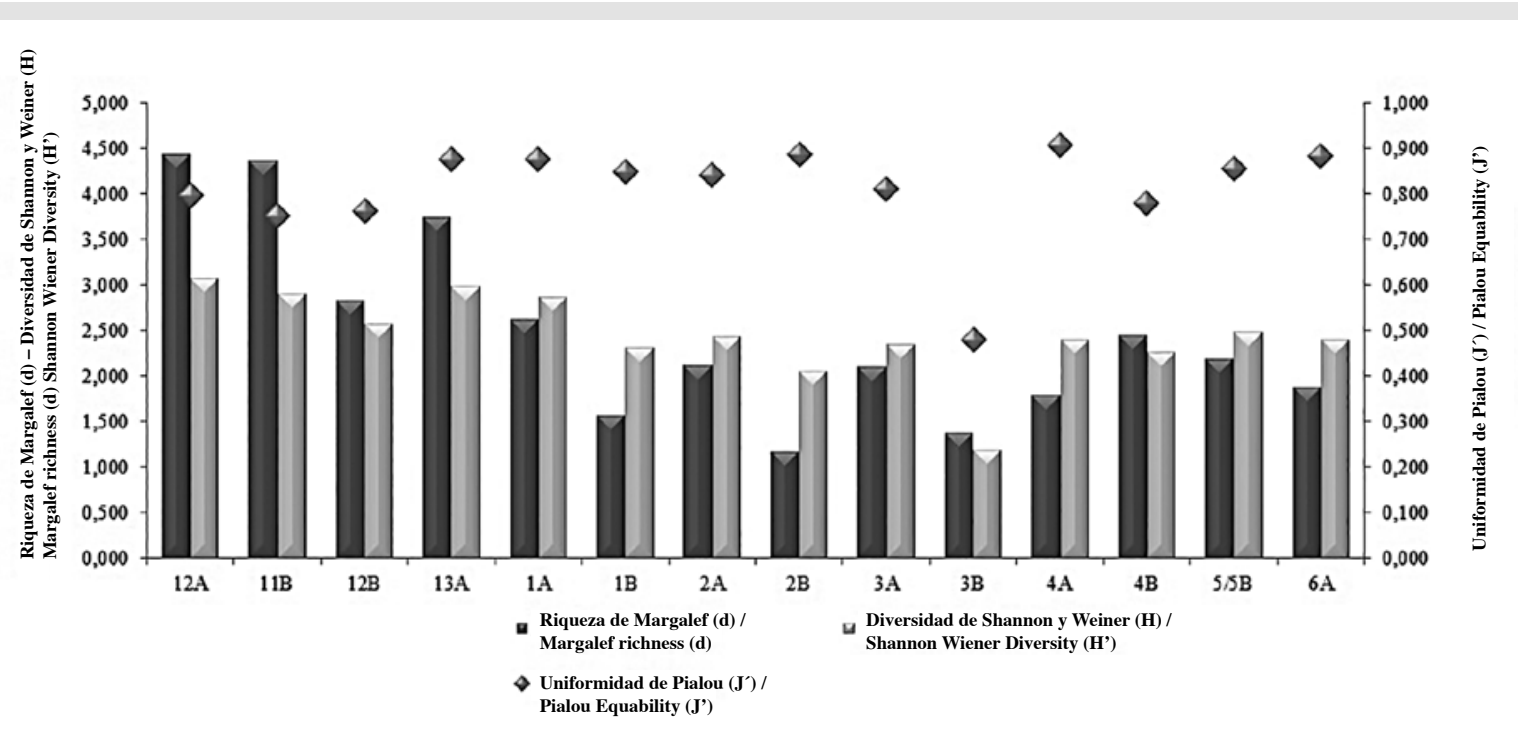

Figura 4. Comportamiento de los índices ecológicos encontrados en la grilla de estaciones, durante la III Expedición Científica de Colombia a la Antártica “Almirante Padilla" Verano Austral 2016-2017

Figure 4. Behavior of the ecological indices found in the stations grid, during the III Colombian Scientific Expedition to the Antarctic "Almirante Padilla" Austral Summer 2016-2017.
3,074 y 1,193; así mismo, la riqueza se muestra más alta en las estaciones del Pacífico presentando el máximo en la estación 12A con 4,4 mientras que el mínimo se registró en una estación del polo sur (2,5 en la estación 2B).

En el análisis de clasificación se observaron cuatro agrupamientos (Figura 6). El grupo 1 está representado solamente por la estación 2A (Antártica), que presenta la característica espacial de estar más al sur de los demás puntos de muestreo en la Antártica (Figura 2), además de presentar baja densidad de organismos (3015 ind $\bullet 100 \mathrm{~m}^{-3}$ ), donde la mayor abundancia fue representada por la familia Calanidae con 591 ind $100 \mathrm{~m}^{-3}$. El grupo 2 está representado principalmente por el resto de estaciones de la Antártica y la estación más meridional del Pacífico (13A) con una similaridad entre 50 y $70 \%$. El grupo 3 representa una similaridad de $60 \%$ aproximadamente y comprende dos estaciones ubicadas en el Estrecho de Gerlache. Estas dos estaciones se relacionan por su baja densidad. El grupo 4 alcanza una similaridad de $60 \%$ y abarca las estaciones restantes del Pacífico sur, caracterizadas por presentar altas densidades by high densities and high diversity (Figure 6). In these 3 stations high densities are influenced by the great abundance of copepods of the Calanidae family, as observed in station $11 \mathrm{~B}$ with 6057 ind $\bullet 100 \mathrm{~m}^{-3}$ (Table 1 , Figure 3 ).

In order to respond to the hypothesis of finding the degree of similarity and/or connectivity between the South Pacific and Antarctica, statistical tests were carried out to confirm whether there is a greater/lesser abundance of zooplankton in the stations sampled in the Strait of Gerlache compared to the Pacific. The starting point was the conformation of the marine current system, in which the Antarctic Circumpolar Current (ACC) constitutes an important element for global deep circulation, because it transports intermediate and deep water between the Pacific, Atlantic and Indian oceans. The ACC is therefore an important part of the global ocean transport network by redistributing heat around the Earth. With the above, it would be expected that the zooplanktonic community, when presenting great influence in its movement by marine 


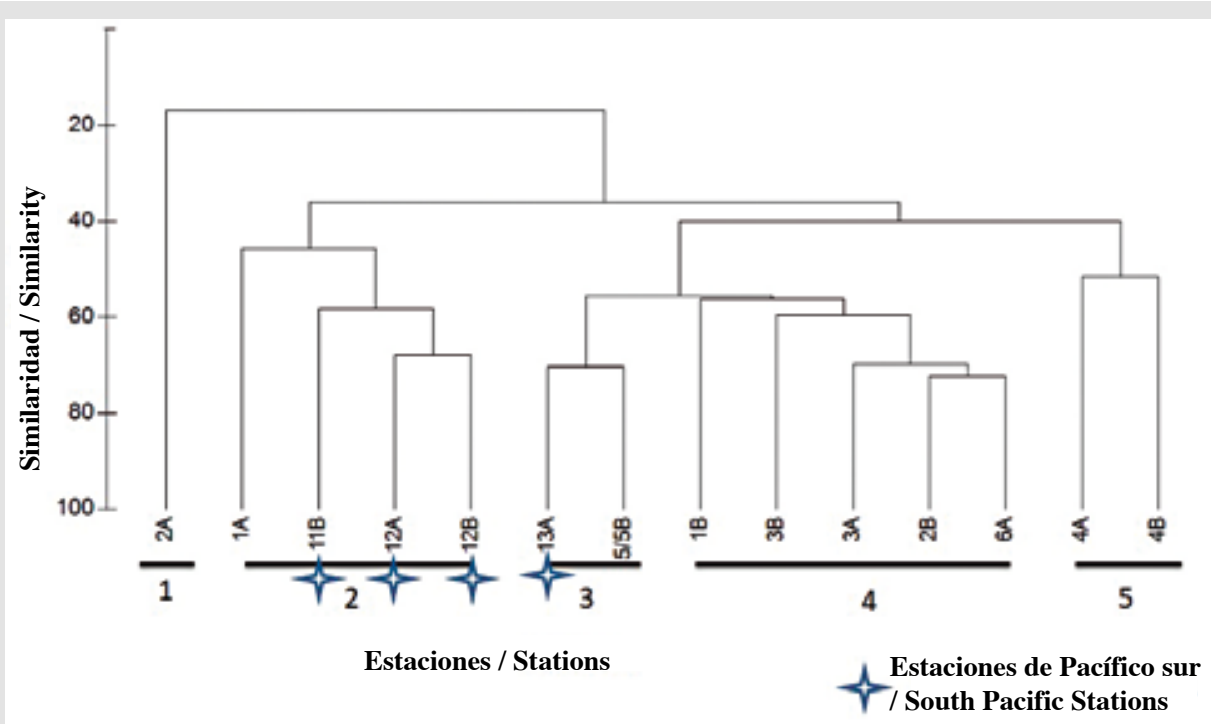

Figura 5. Dendrograma de Similaridad de Bray-Curtis para las estaciones de muestreo en el Pacífico sur y el Estrecho de Gerlache. Índice cofenético: 0,95.
Figure 5. Bray-Curtis Similarity Dendogram for sampling stations in the South Pacific and Gerlache Strait. Co-phenaetic index: 0.95. y diversidad elevada (Figura 6); en estas tres estaciones las altas densidades están influenciadas por la gran abundancia de copépodos de la familia Calanidae, como se observa en la estación 11B con 6057 ind•100 m²(Tabla 1,Figure 5).

Con el fin de responder a la hipótesis de encontrar el grado de similaridad y/o conectividad entre el Pacífico Sur y La Antártica, se realizaron pruebas estadísticas para confirmar si en las estaciones muestreadas en el Estrecho de Gerlache existe mayor/menor abundancia de zooplanctóntes en comparación al Pacífico. Se partió de la conformación del sistema de corrientes marinas, en la que la Corriente Circumpolar Antártica (CCA) constituye un elemento importante para la circulación profunda global, debido a que transporta agua intermedia y profunda entre los océanos Pacífico, Atlántico e Índico. Por lo tanto, la CCA es una parte importante de la red mundial del transporte oceánico redistribuyendo el calor alrededor de la Tierra. Con lo anterior se esperaría que la comunidad zooplanctónica al presentar gran influencia en su movimiento por las corrientes marinas, aparentemente con alta productividad, fuera mayor en esta zona polar. Se definió un $\mathrm{K}=2$, considerando por una parte las densidades totales de las estaciones de la Antártica y por otra aquellas de las estaciones del Pacífico Sur. No obstante, no se cumplieron los supuestos de normalidad y homogeneidad de varianzas, a partir de la prueba ShapiroWilk (Antártica: 0,69, valor-p $\leq$ 0,01; Pacífico Sur: 0,94, valor-p $=0,62, \alpha=0,05$ ) y la prueba de Levene (L: 11,75, valor-p $\leq 0,01)$. Por lo anterior, se realizó la prueba no currents, apparently with high productivity, would be greater in this polar zone. $\mathrm{A} \mathrm{K}=2$ was defined, considering on the one hand the total densities of the Antarctic stations and on the other, those of the South Pacific stations. However, based on the Shapiro-Wilk test (Antarctica: 0.69, p-value $\leq 0.01$; South Pacific: 0.94, p-value $=0.62, \alpha=0.05$ ) and the Levene's test (L: $11.75, \mathrm{p}$-value $\leq 0.01)$ the assumptions of normality and homogeneity of variances were not met. As a result, Mood's nonparametric test was performed, finding that the densities of the zooplankton community in Antarctica are lower than those of the South Pacific (Mood: 1.4 , value $-\mathrm{p}: 0.24, \alpha=0.05)$.

\section{DISCUSSION}

This validates that this group is characterized by inhabiting the pelagic zone of all aquatic ecosystems in the world, where they can predominate in number and biomass, constituting between 60 and $80 \%$ of zooplankton (Campos and Suárez-Mórales, 1994; Boltovskoy, 1999). Therefore, their role in trophic plots and in the vertical flow of carbon is increasingly recognized (López and Mojica, 2015). Likewise, they are reported in studies carried out in the South Pole where the frequency of appearance of copepods in zooplanktonic samples is $100 \%$ (Bonicelli et al., 2008). Most of the families of copepods found in this study have been recorded for both the South Pacific and the Antarctic continent (Bonicelli et al., 2008; López, 2012; Pascal, 
paramétrica de Mood, encontrando que las densidades de la comunidad zooplanctónica en la Antártica son menores que las del Pacífico Sur. (Mood: 1,4, valor -p: 0,24, $\alpha=0,05$ ).

\section{DISCUSIÓN}

Los copépodos fue el grupo que mayor aportó en la composición de esta comunidad sin importar la latitud de los puntos de muestreo, lo que valida que este grupo se caracteriza por habitar la zona pelágica de todos los ecosistemas acuáticos del mundo, donde pueden predominar en número y biomasa, llegando a constituir entre 60 y $80 \%$ del zooplancton (Campos y Suárez-Mórales, 1994; Boltovskoy, 1999). Por lo tanto, su rol en las tramas tróficas y en el flujo vertical de carbono es cada vez más reconocido (López y Mojica, 2015). Así mismo, se registran en estudios realizados en el polo sur donde la frecuencia de aparición de copépodos en las muestras zooplanctónicas es de $100 \%$ (Bonicelli et al., 2008). La mayoría de las familias de copépodos encontrados en esta investigación han sido registradas tanto para el Pacífico sur como para el continente Antártico (Bonicelli et al., 2008; López, 2012; Pascal, 2012; Jaimes y López, 2013; Medellín- Mora, 2016; Jeréz-Guerrero et al., 2017; DoradoRoncancio, 2018). Este estudio demuestra de nuevo que los copépodos son los organismos metazoarios más numerosos del planeta (Longhurst, 1985) y están considerados como el mayor componente de la biomasa mesozooplanctónica del océano Sur (Boysen-Ennen et al., 1991). Las principales familias están representadas por Oithonidae, Oncaeidae, Pseudocalanidae, Calanidae y Metrididae (Cabal et al., 2002). La comunidad zooplanctónica que se distribuye desde el Pacífico sur hasta la Antártica, con base a su composición y densidad de organismos, se considera típica de aguas neríticasoceánicas, presentando dominancia de clases y familias como calanoideos (Calanidae y Paracalanidae) y poecilostomatoides (Oncaeidae), los cuales han sido ampliamente registrados para la Península Antártica (Bonicelli et al., 2008; Pascal, 2012). A este nivel de resolución taxonómica se puede ver una clara interconectividad entre las áreas de muestreo; adicionalmente, cabe resaltar que el patrón de corrientes que se distribuye por la región genera una distribución de los integrantes zooplanctónicos en diferentes densidades, pero manteniendo similar riqueza, lo que a su vez se refleja en el dendrograma de similaridad de Bray-Curtis. Esta interconectividad es debida principalmente a la Corriente Circumpolar Antártica, elemento primordial para circulación global, porque es capaz de transportar agua intermedia y profunda de los océanos
2012; Jaimes and López, 2013; Medellín-Mora, 2016; Jeréz-Guerrero et al., 2017; Dorado- Roncancio, 2018). This study again demonstrates that copepods are the most numerous metazoan organisms on the planet (Longhurst, 1985) and are considered to be the largest component of the mesozooplankton biomass of the Southern Ocean (Boysen-Ennen et al., 1991). The main families are represented by Oithonidae, Oncaeidae, Pseudocalanidae, Calanidae and Metrididae (Cabal et al., 2002). The zooplanktonic community that is distributed from the South Pacific to Antarctica, based on its composition and density of organisms, is considered typical of neriticoceanic waters, presenting dominance of classes and families such as calanoids (Calanidae and Paracalanidae), and poecilostomatoids (Oncaeidae), which have been widely reported for the Antarctic Peninsula (Bonicelli et al., 2008; Pascal, 2012). At this level of taxonomic resolution, it is possible to see a clear interconnectivity between the sampling zones, and it is necessary to emphasize that the pattern of currents that is distributed by zone, generates a distribution of the zooplanktonic members in different densities, but maintaining similar richness, which in turn is reflected in the Bray-Curtis similarity dendogram. This interconnectivity is mainly due to the Antarctic Circumpolar Current, a primordial element for global circulation, because it transports intermediate and deep water from the Pacific, Atlantic, and Indian oceans. It also contributes to circulation in all the basins, and in the specific case of the Pacific from the Chile-Peru or Humboldt Current (Figure 4) that originates a homogeneous distribution of families from high latitudes to subtropical and tropical zones of this oceanic basin.

The Perciformes order was representative in this study showing $12 \%$ relative importance. The families that are grouped in this order are found in larval stages with different levels of swimming capacity and are therefore distributed in several strata of the water column (Zhou et al., 1994; Beltran-León and Rios, 2000). Most of these fish are of diurnal habits, which explains the appearance of several of their larvae in this study, mainly because there is constant sunlight on the white continent during the austral summer, which tends to favor the autotrophic organisms of the plankton, thus generating a high food supply for the zooplankton. The latter is the food resource that sustains the ichthyoplankton in this area. The lowest abundance in percentage was found in the orders Poecilostomatoides and Aulopiformes. The first are small-size copepods that 
Pacífico, Atlántico e Índico. Además, contribuye con la circulación en todas las cuencas y, en el caso específico del Pacífico, a partir de la corriente de Chile-Perú o de Humboldt (Figura 4) originar una distribución homogénea de familias desde alta latitudes hasta áreas subtropicales y tropicales de esta cuenca oceánica.

El orden Perciformes fue representativo en este estudio mostrando un $12 \%$ de importancia relativa. Las familias que se agrupan en este orden se encuentran en estadios larvales con diferentes niveles en la capacidad de nado y por lo tanto se distribuyen en varios estratos de la columna de agua (Beltran-León y Rios, 2000; Zhou et al., 1994). La mayoría de estos peces son de hábitos diurnos, lo que explica la aparición de varias de sus larvas en este estudio, principalmente porque en el continente blanco durante el verano austral siempre hay luz solar, lo cual suele favorecer a los organismos autótrofos del plancton, generando así alta oferta alimenticia para el zooplancton que resulta ser el recurso alimentario que sostiene el ictioplancton en esta zona. La menor abundancia en porcentaje se encontró en los órdenes Poecilostomatoida y Aulopiformes. El primero lo constituyen copépodos de tallas pequeñas que se han descrito para el Pacífico sur y la Antártica (Schnack-Schial et al., 2010; López, 2012; Pascal, 2012; Jaimes y López, 2013).

De otra parte, diferentes estudios realizados en el Pacífico sur, registran entre 39 y 76 familias/morfotipos (López, 2012, Jaimes y López, 2013), con la riqueza estrechamente relacionada con la época de muestreo; suelen encontrarse comunidades más ricas en época seca, principalmente en aguas abiertas donde la incidencia de los eventos de surgencia suelen ser mayores en comparación a los costeros (Franco- Herrera, 2005); la surgencia favorece el aporte de nutrientes a la columna de agua. Particularmente el continente Antártico es considerado por muchos autores como una región muy productiva para todos los niveles tróficos y también en un área altamente influenciada por eventos de surgencias (Huntley et al., 1990; Loeb et al., 1993; Zhou et al., 1994; Van der Molen, 2003). Sin embargo, al realizar estadística inferencial, para confirmar que allí debería haber mayor densidad del zooplancton, se encontró lo contrario. Por lo tanto, se podría indicar que en la Antártica existen otros factores que pueden influenciar fuertemente la productividad; para estas áreas se han descrito que en algunos puntos se presentan zonas altas en nutrientes pero bajas en clorofila (HNLC, por sus siglas en inglés; Pascal, 2012). Estas zonas son consideradas muchas veces como críticas debido que se estarían afectando directamente los have been described for the South Pacific and Antarctica (Schnack-Schial et al., 2010; López, 2012; Pascal, 2012; Jaimes and López, 2013).

On the other hand, different studies carried out in the South Pacific report between 39 and 76 families/ morphotypes (López, 2012, Jaimes and López, 2013), where richness is closely related to the sampling season. Richer communities tend to be found in the dry season, mainly in open waters where the incidence of upwelling events tend to be higher compared to coastal ones (Franco-Herrera, 2005). Upwelling favors the contribution of nutrients to the water column. In particular, the Antarctic continent is considered by many authors as a very productive zone for all trophic levels and also an area that is highly influenced by upwelling events (Huntley et al., 1990; Loeb et al., 1993; Zhou et al., 1994; Van der Molen, 2003). However, when inferential statistics were used to confirm that there should be a higher density of zooplankton, the opposite was found. Therefore, it could be indicated that on the white continent there are other factors that can strongly influence productivity. For these Antarctic zones it has been described that in some parts, there are areas high in nutrients but low in chlorophyll (HNLC; Pascal, 2012). These zones are often considered critical, because they directly affect the highest trophic levels of the food web, while the low temperature conditions of Antarctica force the different species to adopt survival strategies (Quetin and Ross, 2001). The amount of light and stability of the water column have a direct influence on the planktonic communities. UV-B radiation also directly affects primary productivity as it inhibits the growth of phytoplankton by up to $90 \%$ (Llabrés and Agustí, 2006). These sub-Antarctic zones have been described as vulnerable to the hole in the ozone layer due to their proximity during the summer. In addition the reflection of these rays on the ice in this subpolar zone and the water contributes to the high levels of UV radiation exposure, which forces phytoplanktonic adaptations or, on many occasions, inhibits productivity in the area (Hernando, 2016). This explains the low composition and abundance of zooplankton in these areas, given the alterations in their food supply, possibly due to this radiation exposure mentioned above.

Zooplankton is in the second link of the marine food web, well represented by copepods and krill, which happen to be the direct consumers of phytoplankton (first trophic link). Although Antarctica is the most isolated continent, it is not free of environmental and anthropogenic pollution, demonstrated by the high heavy metal contents 
niveles tróficos más altos de la red alimentaria, también las condiciones de temperaturas bajas de la Antártica obligan a las diferentes especies a tener estrategias de supervivencia (Quetin y Ross, 2001). La cantidad de luz y estabilidad de la columna de agua influyen directamente en las comunidades planctónicas; también la radiación ultravioleta UV-B afecta directamente la productividad primaria, ya que inhibe el crecimiento del fitoplancton hasta 90\% (Llabrés y Agustí, 2006). Estas áreas sub-Antárticas se han descrito como vulnerables frente al agujero en la capa de ozono, por su cercanía durante el verano; además el reflejo de estos rayos sobre el hielo de esta zona subpolar y el agua contribuye a los elevados niveles de exposición de radiación UV, lo que obliga a adaptaciones fitoplanctónicas o en muchas ocasiones inhibe la productividad en la zona (Hernando, 2016). Esto da respuesta a la baja composición y abundancia del zooplancton en estas áreas por las alteraciones en la oferta alimentaria, posiblemente por esta radiación.

El zooplancton está ubicado en el segundo eslabón de la red trófica marina, que es bien representado por los copépodos y el krill, que resultan ser los consumidores directos del fitoplancton (primer eslabón trófico). Pese a que la Antártica es el continente más aislado, no está libre de contaminación, tanto ambiental como antropogénica, por tal motivo se han detectado en estas áreas altos contenidos de metales pesados (Ribeiro et al., 2011; Sarmiento y Flores, 2014). Estos factores fisicoquímicos pueden explicar también las bajas densidades de organismos zooplanctónicos en las estaciones antárticas muestreadas, pues podrían estar afectando no solo la red trófica marina, sino la industria pesquera y en general toda la dinámica ecosistémica de estas áreas.

Es preciso destacar algunas estrategias de la comunidad zooplanctónica, las cuales explican las diferencias en las densidades de organismos en las estaciones muestreadas. La migración vertical que realizan algunos de los integrantes del zooplancton (Bo-Ping y Straskraba, 1998; Boxshall y Halsey, 2004), desplazándose a la superficie en el día por la influencia de factores abióticos (Bollens y Frost, 1989), ocasiona cambios de densidades en el momento del muestreo. Este proceso migratorio puede variar según la especie, el tamaño y estado de desarrollo (Aveces et al., 2007).

La aparición de la familia Sagittidae en este estudio, y en general de organismos gelatinosos, son concordantes a lo registrado para áreas antárticas (Bonicelli et al., 2008; IAC, 2008; Pascal, 2012). La presencia del phylum Chaetognatha, that have been detected here (Ribeiro et al., 2011; Sarmiento and Flores, 2014). These physicochemical factors may also explain the low densities of zooplanktonic organisms in the sampled Antarctic stations, and could be affecting not only the marine food web, but also the fishing industry and, in general, the entire ecosystem dynamics of these areas.

It is important to highlight some of the strategies used by the zooplankton community, which explain the differences in the densities of organisms in the sampled stations. The vertical migration of some of the zooplankton members (Bo-Ping and Straskraba, 1998; Boxshall and Halsey, 2004), which move to the surface during the day due to the influence of abiotic factors (Bollens and Frost, 1989), causes density changes at the time of sampling. This migratory process may vary according to species, size and stage of development (Aveces et al., 2007).

The appearance of the Sagittidae family in this study, and in general of gelatinous organisms, is consistent with reports for Antarctic zones (Bonicelli et al., 2008; IAC, 2008; Pascal, 2012). The presence of Phylum Chaetognatha, which includes gelatinous organisms with primary functions within the zooplankton community, is associated with biological changes (availability of food and degree of predation) (Pascal, 2012). These zooplanktonic gelatinous organisms are distributed from tropical to subarctic and sub Antarctic waters and under favorable conditions salps can proliferate rapidly to form dense aggregations and thus dominate zooplankton biomass (Hereu et al., 2006). In plankton studies in countries such as Costa Rica, large quantities of these gelatinous organisms are also reported as bio indicators of food availability (Castellanos-Osorio et al., 2012), confirming their high density also in Pacific waters.

In this study, decreasing taxa and typical zooplankton organisms such as copepods for seasons 4A and 4B (Antarctica) was notable, mainly due to the predominance of gelatinous organisms, not only from the Sagittidae family, but also salps of the Class Thaliacea. The latter are typical of South Pole waters and in the present study reached densities of up to 281.62 ind $100 \mathrm{~m}^{-3}$. In recent years, gelatinous zooplankton has become an important object of study and its ecological significance in ecological processes has been reconsidered (Mills, 1995). These organisms are active predators within the zooplankton, which explains the low amount of other taxa, especially calanoid copepods in these seasons (López, 2012). In this study, we expected to find a large amount of krill in the sampled stations in the South Pole, represented mainly by the Euphausiidae family, which 
que incluye organismos gelatinosos con funciones primordiales dentro de la comunidad zooplanctónica, se asocia con cambios biológicos (disponibilidad de alimento y grado de depredación) (Pascal, 2012). Estos organismos gelatinosos del zooplancton se distribuyen desde aguas tropicales a subárticas y subantárticas y en condiciones favorables las salpas pueden proliferar rápidamente llegando a formar densas agregaciones y así dominar la biomasa zooplanctónica (Hereu et al., 2006). En estudios del plancton en países como Costa Rica también se registran grandes cantidades de estos organismos gelatinosos, como bioindicadores de disponibilidad de alimento (CastellanosOsorio et al., 2012), lo que confirma su alta densidad también en aguas del Pacífico.

En este estudio, fue notable la disminución de taxa y de organismos típicos del zooplancton como los copépodos, para las estaciones 4A y 4B (Antártica), debido principalmente a que predominaron los organismos gelatinosos, no solo de la familia Sagittidae, sino también salpas de la clase Thaliacea. Estos últimos resultan ser típicos de aguas del polo sur y alcanzaron en el presente estudio densidades de hasta 281,62 ind $100 \mathrm{~m}^{-3}$. El zooplancton gelatinoso se ha convertido en los últimos años en un importante objeto de estudio y se ha replanteado su significado ecológico en los procesos ecológicos (Mills, 1995). Estos organismos son depredadores activos dentro del zooplancton, lo cual explica la baja cantidad de otros taxa en especial copépodos calanoideos en estas estaciones (López, 2012). Es este estudio se esperaba encontrar gran cantidad de krill en las estaciones muestreadas en el polo sur, representados principalmente por la familia Euphausiidae, que resulta tener una gran importancia dentro de la trama trófica marina, pero en general fueron muy pocas estaciones las que presentaron altas densidades de esta familia (Tabla 1), similar a lo que ocurrió en el verano austral de 2005, cuando la alta presencia de organismos gelatinosos como las salpas sugiere elevada competencia y/o depredación, lo cual se refleja en bajas densidades del krill antártico (Pascal, 2012).

Sin embargo, en el presente estudio se observó en varios sectores de la Península Antártica una distribución del mesozooplancton que sugiere la presión depredadora sobre los copépodos por parte del krill. La distribución del krill de pequeño tamaño presentó un gradiente inverso a la de los copépodos. Es decir, las estaciones con mayor densidad de krill juvenil mostraron una menor densidad de copépodos (Tabla 1). Debido a ello, se puede pensar que estos tiene preferencias alimentarias sobre los copépodos de mayor tamaño, lo cual es is of great importance within the marine food web. However, in general, there were very few stations that presented high densities of this family (Table 1) as occurred in the austral summer of 2005, where the high presence of gelatinous organisms such as salps, suggests high competition and/ or predation reflected in the low densities of Antarctic krill (Pascal, 2012).

However, in the present study, a distribution of mesozooplankton has been observed in several sectors of the Antarctic Peninsula, suggesting predatory pressure on copepods by krill. The distribution of small krill had an inverse gradient to that of copepods. That is, the seasons with the highest density of juvenile krill showed a lower density of copepods (Table 1). Because of this, it may be considered that these have food preferences over the larger copepods, which is similar to reports by Bonicelli et al., (2008) and Pascal, (2012). This indicates that the decreased number of copepods in the Antarctic areas is directly influenced by the predation not only of krill, but also of gelatinous organisms. Those seasons with higher densities of copepods in Antarctica (1A, 1B; Table 1) seem to be associated with thawing zones, which generate a supply of nutrients and cause greater availability of phytoplankton (food supply). The increase of nutrients favors the development of cocolithophorids, diatoms and dinoflagellates that are the favorite food for zooplankton (Sato et al., 2008), and provides them with the appropriate nutritional benefits for sustaining and increasing the richness of this community. The dynamics found from the applied ecological indices, reflects a community that, despite presenting a high representation of copepods, exhibits a high uniformity, which is typical in zooplanktonic communities (López, 2012; Pascal, 2012). The similarity in general terms is high, presenting only certain spatial differences caused by the appearance of gelatinous organisms or by changes in the density of the cosmopolitan groups found.

As already mentioned in this study, the density and richness of zooplankton depend on physicochemical and ecological factors. The main tendency was to find individuals of the families Calanidae, Eucalanidae, Paracalanidae and Sagittidae. In relation to the first three copepods, it is very likely that this sighting is related to food availability in the areas and food choice of these individuals (Saiz and Calbet, 2011), as has been explain in studies on trophic aspects in Mexico (López and Palomares 2006; Lavaniegos et al., 2012) and Chile (Böttjer et al., 2010). The Calanidae family is characterized by consuming fine particles of decaying 
similar a lo registrado por Bonicelli et al.(2008) y Pascal (2012); esto indica que la disminución de copépodos en las zonas antárticas está directamente influenciada por la depredación no solo del krill, sino también de los organismos gelatinosos. Aquellas estaciones con mayores densidades de copépodos en la Antártica (1A, 1B; Tabla 1) parecen estar asociadas a las zonas de deshielo, lo que genera un aporte de nutrientes y que ocasiona una mayor disponibilidad en fitoplancton (oferta alimentaria).El aumento de nutrientes favorece el desarrollo de cocolitofóridos, diatomeas y dinoflagelados que son el alimento predilecto del zooplancton (Sato et al., 2008), lo que proporciona los beneficios nutricionales apropiados para el sostenimiento y aumento de la riqueza de esta comunidad. La dinámica encontrada a partir de los índices ecológicos aplicados refleja una comunidad que, si bien posee una alta representatividad de copépodos, presenta una uniformidad elevada, lo cual resulta ser típico en comunidades zooplanctónicas (López, 2012; Pascal, 2012). La similaridad en términos generales es alta, presentando solo ciertas diferencias espaciales causadas por la aparición de organismos gelatinosos o bien por cambios en la densidad de los grupos cosmopolitas encontrados.

Comoyasehamencionadoenesteestudio,ladensidad y riqueza de los zooplánctones depende de factores fisicoquímicos y ecológicos. La tendencia principal fue encontrar a individuos de las familias Calanidae, Eucalanidae, Paracalanidae y Sagittidae. Con relación a las tres primeras que pertenecen a los copépodos, es muy probable que este avistamiento se relacione con la disponibilidad de alimento en las áreas y la elección alimentaria de estos individuos (Saiz y Calbet, 2011), como se ha tratado de explicar en estudios sobre aspectos tróficos en México (López y Palomares 2006; Lavaniegos et al., 2012) y Chile (Böttjer et al., 2010). La familia Calanidae se caracteriza por consumir partículas finas de fitoplancton en descomposición y probablemente poseer hábitos omnívoros (Boxshall y Halsey, 2004). De forma similar, la familia Eucalanidae se alimenta de los productores primarios por filtración de pequeñas partículas de las zonas epi y mezopelágica en aguas oceánicas- neríticas y suele dominar en el plancton debido a su pequeño tamaño (Boxshall y Halsey, 2004). La familia Paracalanidae presenta hábitos herbívoros y omnívoros. Esta familia puede compartir los recursos alimentarios, pero para minimizar la competencia se distribuye en diferentes capas en la columna de agua, acorde con la termoclina, como estrategia para disminuir la competencia por nicho (Fragopoulu et al., 2001); esta familia domina en ambientes oligotróficos tropicales (Schnack-Schiel et al., 2010). phytoplankton and probably possessing omnivorous habits (Boxshall and Halsey, 2004). Similarly, the Eucalanidae family feeds on primary producers by filtering small particles from the epipelagic and mesopelagic zones in oceanic-neritic waters and tends to dominate in the plankton due to its small size (Boxshall and Halsey, 2004). The Paracalanidae family has both herbivorous and omnivorous habits and may share food resources. However, to minimize competition it is distributed in different layers in the water column, according to the thermocline, as a strategy to decrease niche competition (Fragopoulu et al., 2001). The Paracalanidae family dominates tropical oligotrophic environments (Schnack-Schiel et al., 2010).

\section{CONCLUSIONS}

With this study connectivity was found between the study areas, because statistically the stations are correlated and families typically representative of zooplankton at cosmopolitan level are found in both environments. However, this is not related to the global current system, since we expected to find greater abundance and diversity in waters of the South Pole, where theoretically, all currents converge.

The group that predominated in general was that of copepods, which are still the most representative in the zooplankton samples, mainly the Calanoids, for both the South Pacific and Antarctica.

There was little evidence of krill, which would probably be associated with physicochemical changes in water bodies, which may be related to global climate change that is affecting the dynamics and distribution of these crustaceans.

Large quantities of gelatinous organisms were found in some Antarctic stations, especially salps, which could be related to strong predatory pressure against krill and copepods, which would affect zooplanktonic dynamics.

The low abundance of zooplankton in Antarctica, demonstrate possible changes and effects on the development of fisheries and the planet's biodiversity. For this reason, more studies are needed that focus on the life strategies of zooplankton in the face of these changes, as well as on the identification of food habits, heavy metals, stable isotope molecular biology, among other studies, to help characterize and understand the dynamics of plankton in Antarctica and its relationship with other marine ecosystems a little more clearly. 


\section{CONCLUSIONES}

Con este estudio se encontró conectividad entre las áreas de estudio, debido a que estadísticamente se correlacionan las estaciones y taxonómicamente se encuentras familias típicas en ambos ambientes representativas del zooplancton a nivel cosmopolita; sin embargo, esto no se relaciona al sistema de corrientes globales, puesto que se esperaba encontrar mayor abundancia y diversidad en aguas del polo sur, donde teóricamente convergen todas las corrientes.

El grupo que predominó en general fueron los copépodos, que siguen siendo los más representativos en los muestreos del zooplancton, principalmente los Calanoideos, tanto para el Pacífico sur como para la Antártica.

Se evidenció poca cantidad de krill, lo cual probablemente se asocia con cambios fisicoquímicos en las masas de agua, que pueden relacionarse con el cambio climático global que está afectando la dinámica y distribución de estos crustáceos.

En algunas estaciones de la Antártica se encontraron grandes cantidades de organismos gelatinosos, especialmente salpas, lo cual podría relacionarse como fuerte presión depredadora frente al krill y los copépodos, que estaría afectando la dinámica zooplanctónica.

Las bajas abundancias de zooplancton en la Antártica demuestran posibles cambios y afectaciones hacia el desarrollo en pesquerías y biodiversidad del planeta, para lo cual se necesitan más estudios enfocados hacia las estrategias de vida del zooplancton frente a estos cambios, así como identificación de hábitos alimentarios, metales pesados, biología molecular, isotopos estables, entre otros estudios, que ayuden a caracterizar y entender un poco más la dinámica del plancton en la Antártica y su relación con los otros ecosistemas marinos.

\section{AGRADECIMIENTOS}

Los autores quieren agradecer al Programa Antártico Colombiano que hizo posible la III Expedición Científica de Colombia a la Antártica "Almirante Padilla" (Verano Austral 2016-2017), donde se pudo gracias a un trabajo conjunto aportar información científica por medio de este manuscrito. También se agradece a la Universidad Jorge Tadeo Lozano, Sede Santa Marta, por el acceso a sus instalaciones, préstamo de equipos y materiales para el procesamiento de las muestras, así como a todo el personal de la universidad, trabajadores y profesores, que siempre estuvieron pendientes de nuestros requerimientos.

\section{ACKNOWLEDGEMENTS}

The authors would like to thank the Colombian Antarctic Program that made the III Scientific Expedition of Colombia to the Antarctic "Almirante Padilla" (Summer 2016-2017) which was possible thanks to a joint effort to provide scientific information on Antarctica through this manuscript. Thanks are also due to the Jorge Tadeo Lozano University, Santa Marta Headquarters, for access to its facilities, loan of equipment and materials, for sample processing, as well as to all the university staff, employees and professors who were always ready to deal with any request and/or permission. 


\section{BIBLIOGRAFÍA / LITERATURE CITED}

Anderson, J.B. 1999. Antarctic Marine Geology, Cambridge University Press, 289 p.

APHA (American Public Health Association), AWWA (American Water Works Association) and WEF (Water Environment Federation). 2005. Standard methods for the examination of water and wastewater. $21 \mathrm{ed}$. USA $981 \mathrm{p}$.

Aveces, G., G. Esqueda, R. Pacheco, A. Zárate, J. Hernández y S. Hernández. 2007. Cambios diarios en la composición y abundancia de copépodos planctónicos al sur de la bahía de La Paz (octubre de 2002). Hidrobiológica, 17(2): 185-188.

Bárcena, M., J. Sesma, E. Isla y A. Palanques. 2005. Respuesta del registro sedimentario a la ciclicidad solar en el estrecho de Gerlache (península Antártica). http://rabida.uhu.es/dspace/handle/10272/8756.06/10/2018.

Bernard, K.S y P.W. Froneman. 2002. Mesozooplankton community structure in the Southern Ocean upstream of the Prince Edward Islands. Polar Biol., 25: 597-604

Björberg, T.K.S. 1981. Atlas del zooplankton del Atlántico sudoccidental y métodos de trabajo con el zooplankton marino. Copepoda: 586-679. INIDEP. Argentina.

Blanco, J.L., A.C. Thomas, M.E. Carr and P.T. Strub. 2001. Seasonal climatology of hydrographic conditions in the upwelling region off northern Chile. J. Geophys Res., 106(C6): 11451-11467.

Bollens, S. M. and B. W. Frost, 1989. Predator induced diel vertical migration in a marine planktonic copepod. J. Plankton Res., 11: $1047-1065$.

Boltovskoy, D (Ed.). 1981. Atlas del zooplancton del Atlántico Sudoccidental y métodos de trabajo con el zooplancton marino. Publicación Especial del INIDEP. Mar del Plata, Argentina. 936 p.

Boltovskoy D Ed. 1999. South Atlantic zooplankton. Backhuys Publishers, Leiden, Netherlands,1-2: 1-1706 p.

Bonicelli, J., D. López, N. Ochoa and R. Shreeve. 2008. Zooplankton community structure and its association with the phytoplankton and water masses from the Bransfield strait and Elephant island during 2006 austral summer. Ecol. Apl., 7(1,2): 159-164.

Bo-Ping, H. and M. Straskraba. 1998. Modeling patterns of zooplankton diel vertical migration. J. Plankton Res., 20 (8): 1463 - 1487.

Böttjer, D., C.E. Morales and U. Bathmann. 2010. Trophic role of small cyclopoid copepod nauplii in the microbial food web: a case study in the coastal upwelling system off central Chile. Mar. Biol., 157:689-705.

Boxshall, G. A. and S. H. Halsey. 2004. An introduction to copepod diversity: Part I-II. The Ray Society. London. 966 p.

Boysen-Ennen, E., W. Hagen, G. Hubold and U. Piatkowski. 1991. Zooplankton biomass in the ice covered Weddell Sea, Antarctica. Mar. Biol., 111: 227235 .

Bradford-Grieve, J.M., E.L. Markhaseva, C.E. Rocha and B. Abiahy. 1999. Copepoda: 869-1098. In: Boltovskoy D. (Ed.). South Atlantic zooplankton, Vol. 2. Backhuys Publishers, Leiden, Neteherlands.

Buskey, E. J., P. H. Lenz and D. K. Hartline. 2012. Sensory perception, neurobiology, and behavioral adaptations for predator avoidance in planktonic copepods. Adapt. Behav., 20: 57-66.

Cabal, J. A., F. Álvarez-Marqués, J.L. Acuña, M. Quevedo, R. Gonzalez-Quirós, L. Huskin, D. Fernández, C. Rodríguez Del Valle and R. Anadón. 2002. Mesozooplankton distribution and grazing during the productive season in the Northwest Antarctic Peninsula (FRUELA cruises). Deep-Sea Res. II, 49: 869-881.

Campos- Hernández, A. y E. Suárez-Morales. 1994. Copépodos pelágicos del golfo de México y mar Caribe. I Biología y Sistemática. Centro de investigaciones de Quintana Roo (CIQRO). México. 353 p.

Castellanos-Osorio, I., R. Hernández-Flores., A. Morales-Ramírez y M. Corrales. 2012. Apendicularias (Urochordata) y quetognatos (Chaetognatha) del Parque Nacional Isla del Coco, Costa Rica. Rev. Biol. Trop., 60 (3): 243-255.

CCCP. 2002. Compilación oceanográfica de la Cuenca Pacífica Colombiana. Centro Control de Contaminación del Pacífico, Tumaco., 1-109p p.

CPPS. 2014. Estudio para determinar la contribución de océano Pacífico Suroriental como sumidero de gases de efecto invernadero. Com. Perm. Pacífico Sur- CPPS. Guayaquil. Serie Est. Reg., 3: 2-59.

Dorado-Roncancio, J.H. 2018. Variabilidad de la composición y abundancia de la subclase Copepoda en el océano Pacífico colombiano durante septiembre de 2005 y 2007. Trabajo de grado Biología. Universidad Nacional de Colombia, Bogotá. 86 p.

FAO, 1995. Code of conduct for responsible fisheries. Food and Agriculture Organization, Rome. 41 p.

Fernández, M. 2015. Orden Calanoida. Ibero diversidad entomológica. Idea-sea., 89: 1-27. http://sea-entomologia.org/IDE@/revista_89.pdf. 04/06/2018.

Field, J., K. Clark and R. Warwick. 1982. A practical strategy for analyzing multispecies distribution pattern. Mar. Ecol. Prog. Ser., 8: 37-52 p.

Fragopoulu, N., I. Siokou-Frangou, E.D. Christou and M.G. Mazzocchi. 2001. Patterns of vertical distribution of Pseudocalanidae and Paracalanidae (Copepoda) in pelagic waters (0 to $300 \mathrm{~m}$ ) of the eastern Mediterranean Sea. Crustaceana, 74 (1): 49-68.

Franco-Herrera, A. 2005. Oceanografía de la Ensenada de Gaira- El Rodadero más que un centro turístico en el Caribe colombiano. Univ. Jorge Tadeo Lozano, Santa Marta. 56 p.

García, M.A., C. Castro, G. Ríos, A.F. Doval, M.D. Rosóm, G. Gomis, D. and O. López. 2002. Water masses and distribution of physicochemical properties in the Western Bransfield Strait and Gerlache Strait during Austral summer 1995/96. Deep Sea Res. Part II: Stud. Ocean., 49(4): 585-602. 
González, A. 1988. Plancton de las aguas continentales. Org. Est. Am., Washington, D.C. 130 p.

Hereu, C.M., B.E, Lavaniegos, G. Gaxiola-Castro and M.D. Ohman. 2006. Composition and potential grazing impact of salp assemblages off Baja California during the 1997-1999 El Niño and La Niña. Mar. Ecol. Progr. Ser., 318: 123-140.

Hernando, M. 2016. Efectos de la radiación solar sobre el fitoplancton de aguas antárticas y sub-antárticas. Trabajo de grado. Universidad de Buenos Aires. 295 p. https://www.researchgate.net/publication/277052716_Efectos_de_la_radiacion_solar_sobre_el_fitoplancton_de_aguas_antarticas_y_ subantarticas. 05/04/2019.

Heron, G. and J. Bradford-Grieve. 1995. The marine fauna of New Zealand: Pelagic Copepoda: Poecilostomatoida, Oncaeidae. New Zealand Oceanographic Institute. $72 \mathrm{p}$.

Hopkins, T. L. 1985. The zooplankton community of Croker Passage, Antarctic Peninsula. Pol. Biol., 4:161- 170.

Hosie, G. W. 2000. The macroplankton communities in the Prydz Bay region, Antarctica: 93-123. In: El Sayed, S.Z. (Ed.). Southem Ocean ecology: the BIOMASS perspective. Cambridge University Press, Cambridge. 421p.

Huntley, M.E., E. Brinton, M.D.G. López, A. Townsend and W. Nordhausen. 1990. RACER: Fine-scale and mesoscale zooplankton studies during the spring bloom, 1989. Ant. J. US, 25: 157-159.

IAC. 2008. Instituto Antártico Chileno. Rev. Cienc. Ant. Chil., 27(2):1-40. http://www.inach.cl/inach/wp-content/uploads/2009/10/Boletin-AntarticoDic2008.pdf. 09/06/2018.

INVEMAR. 2017. Mapa estaciones de muestreo notas información. http://www.invemar.org.co/noticias/-/asset_publisher/K9IDHIbBbV40/content/ invemar-presente-en-la-primera-expedicion-de-colombia-a-laAntártica/pop_up?_101_INSTANCE_K9IDHIbBbV40_viewMode=print. 06/06/2018.

Izaguirre, I y R. Sánchez. 2005. Ecorregiones Antártica e Islas del Atlántico Sur. Situación ambiental Argentina. Lab. Limnol., Dep. Ecol. Gen. Evol., Fac. Cienc. Exac. Nat. (FCEN), Universidad de Buenos Aires (UBA). II Direc. Nal Ant.

Jaimes, J. C. y R. H. López. 2013. Biomasa y abundancia de Copepoda (Crustacea) en aguas superficiales del océano Pacífico colombiano durante septiembre de 2007. Rev. Biol. Mar. Oceanogr., 49(1): 31-41.

Jerez-Guerrero, M., C. Hernández y A. Giraldo. 2017. Copépodos epipélagicos en bahía Cupica. Pacífico colombiano: Composición de especies, distribución y variación temporal. Rev. Biol. Trop., 65(3): 1046-1061.

Knox, G. A. 2006. Biology of the Southem Ocean. Publ CRC Press, Boca Raton 621p.

Lavaniegos, B.E., G. Heckel y P.Ladrón De Guevara. 2012. Variabilidad estacional de copépodos y cladóceros de bahía de los Ángeles (golfo de California) e importancia de Acartia clausi como alimento del tiburón ballena. Cienc. Mar., 38(1): 11-30.

Laws, R.M.1985. The ecology of the Southern Ocean. Am. Scient., 73: 26-40.

Llabrés, M. and S. Agustí. 2006. Picophytoplankton cell death induced by uv radiation: evidence for oceanic Atlantic communities. Limnol. Oceanogr., 51:21-29.

Loeb, V.J., A.K. Kellermann, P. Koubbi, A.W. North and M.G. White. 1993. Antarctic larval fish assemblages: a review. Bull. Mar. Sci., 53(2): 416-449.

Loeb, V.J., V. Siegel, O. Holm-Hansen, R. Hewitt, W. Fraser, W. Trivelpiece and S. Trivelpiece. 1997. Effects of sea-ice extent and krill or salp dominance on the Antarctic food web. Nature, 387: $897-900$.

Longhurst, A.R. 1985. Relationship between diversity and the vertical structure of the upper ocean. Deep-Sea Res., 32: 1535-1570.

Lonin, S. 2015. Un modelo hidrodinámico del Estrecho de Gerlache (Antártica) para el verano del hemisferio sur. Bol. Cient. CIOH, 33 : $145-167$.

López, G.A. 2008. Estructura trófica de los copépodos pelágicos en el océano Pacífico oriental tropical. Tesis Ph. D. Inst. Politécn. Nal, Centro Interdisciplinario Cienc. Mar., La Paz, BCS, México. http://www.repositoriodigital.ipn.mx/bitstream/handle/123456789/14274/lopezib2.pdf?sequence=1.22/03/2018.

López, G.A. y R. Palomares. 2006. Estructura de la comunidad de copépodos en bahía Magdalena, México, durante El Niño 1997-1998. Rev. Biol. Mar. Oceanogr., 41(1): 63-76.

López R. H. 2012 “Distribución y abundancia de copépodos pelágicos en el Pacífico colombiano”, Rev. Fac. Cien., 8(1):98-123.

López, R.H. and L.H. Mojica. 2015. Distribution and abundance of Oncaea media and O. venusta (Crustácea: Copepoda) in the Colombian Pacific Ocean during two periods in 2001. Rev. UDCA Act. Div. Cient., 18(1): 197-206.

Marín, V. y G. Olivares. 1999. Estacionalidad de la productividad primaria en bahía Mejillones del Sur (Chile): una aproximación proceso-funcional. Rev. Chil. Hist. Nat., 72: 629-641.

Medellín-Mora, J. 2016. Factores y mecanismo que modulan la estructura comunitaria del mesozooplancton marino: Efecto de la estratificación vertical de la columna de agua.

Tesis Doctor Ocean. Universidad de Concepción, Concepción, Chile. 152 p.

Mills, C.E. 1995. Meduase, Shiphonophores and Ctenophores as planktivorous predators in changing global ecosystems. ICES J.Mar. Sci., 52: 575-581.

Monsalve, B. 1976. Copépodos del Pacífico colombiano, cruceros Pacífico V y VII, Div. Pesq., 18(3,4): 2-9.

Nishida, S. 1985. Taxonomy and distribution of the family Oithonidae (Copepoda: Cyclopoida) in the Pacific and Indian Ocean. Bull. Ocean Res. Inst., Univ. Tokyo, 20: 1-167. 
Pakhomov, E.A., R. Perissinotto, C.D. Mcquaid and P.W. Froneman. 2000. Zooplankton structure and grazing in the Atlantic sector of the Southem Ocean in late austral summer 1993. Part 1. Ecological zonation. Deep-Sea Res., 147: 1663-1686.

Palomares, R., E. Súarez-Morales y S. Hernández-Trujillo. 1998. Catálogo de los copépodos (Crustácea) pelágicos del Pacífico Mexicano. (CICIMAR-IPN). $351 \mathrm{p}$.

Pascal, L. 2012. Biomasa, distribución y metabolismo del zooplancton en aguas de la Península Antártica. Tesis Doctoral, Dep. Biol., Universidad de Las Palmas de Gran Canaria. Las Palmas, España. 183 p.

Quetin, L. B. and R.M. Ross. 2001. Environmental variability and its impact on the reproductive cycle of Antarctic krill. Am. Zool., 41: 74-89.

Ramírez, F.C. y H.W. Mianzan. 2011. Distribución horizontal de los copépodos del mar argentino. En: Boltovskoy, D. (Ed.). Atlas de sensibilidad ambiental de la costa y el mar argentino. http://atlas.ambiente.gov.ar/. 07/06/2018.

Ramírez, G. A. 1999. Ecología aplicada. Diseño y análisis estadístico. Fundación Universidad Jorge Tadeo Lozano, Bogotá. 325 p.

Ribeiro, A., R. Figueira, C. Martins, C. Silva, E. França, M. Bícego, M. Mahiques and R. Montone. 2011. Arsenic and trace metal contents in sediment profiles from the Admiralty Bay, King George Island, Antarctica. Mar. Poll. Bull., 62: 192-196.

Saiz, E y A. Calbet. 2011. Copepod feeding in the ocean: scaling patterns, composition of their diet and the bias of estimates due to microzooplankton grazing during incubations. Hydrobiologia, 666: 181-196.

Santander, E., L. Herrera y J. Pizarro. 2001. Fluctuación diaria del fitoplancton en la capa superficial del océano durante la primavera de 1997 en el norte de Chile (20¹8`S): 1. Biomasa pigmentaria. Rev. Biol. Mar. Oceanogr., 36(2): 141-153.

Sarmiento, D y R. Flores. 2014. Presencia de metales pesados y semimetales en musgo en torno a la Estación Antártica Ecuatoriana Pedro Vicente Maldonado. Quím. Centr., 4(1): 33-39.

Sato, R., Y. Ishibashi, Y. Tamaka, T. Ishimiru y M. Dagg. 2008. Productivity and grazing impact of oikopleura dioica (Tunicata, Appendicularia) in Tokio Bay. J. Plankton Res., 30 (3): 299-309.

Schnack-Schiel, S. B., E. Mizdalski y A. Cornils. 2010. Copepod abundance and species composition in the Eastern subtropical/tropical Atlantic. Deep-Sea Res. II, 57: 2064-2075.

Siegel, V. and V. Loeb. 1995. Recruitment of Antarctic krill Euphausia superba and possible causes for its variability. Mar. Ecol. Progr. Ser., 123: 45-63.

Steenbeek, J., C. Piroddi, M. Coll, J.H. Heymans, S. Villasante and V. Christensen. 2014. Ecopath 30 Years Conference Proceedings: Extended Abstracts. Fish. Centre Res. Rep., 22(3): 1-237.

Taylor, L. 1961. Aggregation, variance, and the mean. Nature, 189: 732-735.

Tejada, G., L. Castro, A. Navarrete, T. Cardona, L. Otero, F. Afanador y W. Pedroza. 2003. Panorama de la contaminación marina del Pacífico colombiano (DIMAR). Centro de Control de Contaminación del Pacífico. San Andrés de Túmaco, Colombia.

Van Der Molen, S. 2003. Estudio sobre la reproducción de Notothenioidei (Pisces: Perciformes) del océano austral. Tesis de Grado. Universidad Autónoma de Barcelona. $37 \mathrm{p}$.

Zar, J.H. 2010. Biostatistical Analysis. 5th Ed. Prentice-Hall. Upper Saddle River, USA. 946 p.

Zhou M., W. Nordhausen and M.E Huntley. 1994. ADCP measurements of the distribution and abundance of Euphausiids near the Antarctic Peninsula in winter. Deep- Sea Res., 41: 1425-1445.

RECIBIDO / RECEIVED: 25/07/2018

ACEPTADO / ACCEPTED: 13/07/2019 